\title{
Maximum Weight Independent Sets and Matchings in Sparse Random Graphs. Exact Results Using the Local Weak Convergence Method
}

\author{
David Gamarnik, ${ }^{1}$ Tomasz Nowicki, ${ }^{1}$ Grzegorz Swirszcz ${ }^{2}$ \\ ${ }^{1}$ Department of Mathematical Sciences, IBM T.J. Watson Research Center, Yorktown \\ Heights, New York 10598; \\ e-mail: gamarnik@watson.ibm.com; nowicki@watson.ibm.com \\ ${ }^{2}$ Warsaw University, Warsaw, Poland; \\ e-mail:swirszcz@us.ibm.com
}

Received 9 December 2003; accepted 10 August 2004; received in final form 8 November 2004

Published online in Wiley InterScience (www.interscience.wiley.com).

DOI 10.1002/rsa.20072

\begin{abstract}
Let $G(n, c / n)$ and $G_{r}(n)$ be an $n$-node sparse random graph and a sparse random $r$ regular graph, respectively, and let $\mathcal{I}(n, r)$ and $\mathcal{I}(n, c)$ be the sizes of the largest independent set in $G(n, c / n)$ and $G_{r}(n)$. The asymptotic value of $\mathcal{I}(n, c) / n$ as $n \rightarrow \infty$, can be computed using the Karp-Sipser algorithm when $c \leq e$. For random cubic graphs, $r=3$, it is only known that $.432 \leq \liminf _{n} \mathcal{I}(n, 3) / n \leq \lim \sup _{n} \mathcal{I}(n, 3) / n \leq .4591$ with high probability (w.h.p.) as $n \rightarrow \infty$, as shown in Frieze and Suen [Random Structures Algorithms 5 (1994), 649-664] and Bollabas [European J Combin 1 (1980), 311-316], respectively. In this paper we assume in addition that the nodes of the graph are equipped with nonnegative weights, independently generated according to some common distribution, and we consider instead the maximum weight of an independent set. Surprisingly, we discover that for certain weight distributions, the $\operatorname{limit} \lim _{n} \mathcal{I}(n, c) / n$ can be computed exactly even when $c>e$, and $\lim _{n} \mathcal{I}(n, r) / n$ can be computed exactly for some $r \geq 1$. For example, when the weights are exponentially distributed with parameter $1, \lim _{n} \mathcal{I}(n, 2 e) / n \approx .5517$, and $\lim _{n} \mathcal{I}(n, 3) / n \approx .6077$. Our results are established using the recently developed local weak convergence method further reduced to a certain local optimality property exhibited by the models we
\end{abstract}

Correspondence to: D. Gamarnik

(c) 2005 Wiley Periodicals, Inc. 
consider. We extend our results to maximum weight matchings in $G(n, c / n)$ and $G_{r}(n)$. For the case of exponential distributions, we compute the corresponding limits for every $c>0$ and every $r \geq 2$. (c) 2005 Wiley Periodicals, Inc. Random Struct. Alg., 27, 000-000, 2005

\section{INTRODUCTION}

Two models of random graphs considered in this paper are a sparse random graph $G(n, c / n)$ and a sparse random regular graph $G_{r}(n)$. The first is a graph on $n$ nodes $\{0,1, \ldots, n-1\} \equiv$ $[n]$, where each potential undirected edge $(i, j), 0 \leq i<j \leq n-1$, is present in the graph with probability $c / n$, independently for all $n(n-1) / 2$ edges. Here $c>0$ is a fixed constant, independent of $n$. A random $r$-regular graph $G_{r}(n)$ is obtained by fixing a constant integer $r \geq 1$ and considering a graph selected uniformly at random from the set of all $r$-regular graphs on $n$ nodes (graphs in which every node has degree $r$ ). A set of nodes $V$ in a graph $G$ is defined to be an independent set if no two nodes of $V$ are connected by an edge. Let $\mathcal{I}(n, c)$ and $\mathcal{I}(n, r)$ denote the maximum cardinality of an independent set in $G(n, c / n)$ and $G_{r}(n)$ respectively. Suppose the nodes of a graph are equipped with some nonnegative weights $W_{i}, 0 \leq i \leq n-1$ which are generated independently according to some common distribution $F_{w}(t)=\mathbb{P}\left(W_{i} \leq t\right), t \geq 0$. We denote by $\mathcal{I}_{w}(n, c), \mathcal{I}_{w}(n, r)$ the maximum weight of an independent set in $G(n, c / n)$ and $G_{r}(n)$, respectively.

A matching is a set of edges $A$ in a graph $G$ such that every node is incident to at most one edge in $A$. Let $\mathcal{M}(n, c)$ and $\mathcal{M}(n, r)$ denote the maximum cardinality of a matching in $G(n, c / n)$ and $G_{r}(n)$, respectively. It is known that $G_{r}(n), r \geq 3$ has a full matching w.h.p., that is $\mathcal{M}(n, r)=n / 2(\lfloor n / 2\rfloor$ for odd $n)$ w.h.p. [16]. If the edges of the graph are equipped with some nonnegative random weights, then we consider instead the maximum weight of a matching $\mathcal{M}_{w}(n, c)$ and $\mathcal{M}_{w}(n, r)$ in graphs $G(n, c / n), G_{r}(n)$, respectively. The computation of $\mathcal{I}_{w}(n, c), \mathcal{I}_{w}(n, r), \mathcal{M}_{w}(n, c), \mathcal{M}_{w}(n, r)$ in the limit as $n \rightarrow \infty$ is the main subject of the present paper.

The asymptotic values of $\mathcal{M}(n, c)$ for large $n$ and for all constants $c$ were obtained by Karp and Sipser using a simple greedy type algorithm in [17]. The result extends to $\mathcal{I}(n, c)$ but only when $c \leq e$. It is an open problem to compute the corresponding limit for independent sets for the case $c>e$. Likewise it is an open problem to compute the corresponding limit in random regular graphs, or even to show that such a limit exists [3].

The developments in this paper show that, surprisingly, proving the existence and computation of the $\operatorname{limits}_{\lim } \mathcal{I}(n, \cdot) / n, \lim _{n} \mathcal{M}(n, \cdot) / n$ is easier in the weighted case than in the unweighted case, at least for certain weight distributions. In particular, we compute the limits for independent sets in $G_{r}(n), r=2,3,4$ and $G(n, c / n), c \leq 2 e$, when the node weights are exponentially distributed, and we compute the limits for matchings in $G_{r}(n)$ and $G(n, c / n)$ for all $r, c$, when the edge weights are exponentially distributed. It was shown earlier by the first author [14] that the $\operatorname{limit} \lim _{n} \mathcal{M}(n, \cdot) / n$ exists for every weight distribution with bounded support, though the nonconstructive methods employed prevented the computation of the limits.

Our method of proof is based on a powerful local weak convergence method developed by Aldous [1,2], Aldous and Steele [5], Steele [23], further empowered by a certain local optimality property derived in this paper. Local weak convergence is a recursion technique based on fixed points of distributional equations, which allows one to compute limits of some random combinatorial structures (see Aldous and Bandyopadhyay [4] for a recent survey on applications of distributional equations, and Aldous and Steele [5] for a survey on the 
local weak convergence method). In particular, the method is used to compute maximum weight matching on a random tree, when the weights are exponentially distributed. The tree structure was essential in [5] for certain computations and the approach does not extend directly to graphs like $G(n, c / n)$ with $c>1$, where the convenience of a tree structure is lost due to the presence of a giant component. It was conjectured in [5] that a some long-range independence property might be helpful to deal with this difficulty. The present paper partially answers this qualitative conjecture in a positive way. We introduce a certain operator $T$ acting on the space of distribution functions. We prove a certain local optimality property stating that, for example, for independent sets, whether a given node $i$ belongs to the maximum weight independent set is asymptotically independent from the portion of the graph outside a constant size neighborhood of $i$, iff $T^{2}$ has a unique fixed point distribution. Moreover, when $T^{2}$ does have the unique fixed point, the size of the extremal object (say maximum weight independent set) can be derived from a fixed point of an operator $T$. The computations of fixed points is tedious, but simple in principle and the groundwork for that was already done in [5]. We suspect that the long-range independence holds in other random combinatorial structures as well. In fact the issues of long-range independence were already considered by Aldous and Bandyopadhyay [4] and Bandyopadhyay [8] using the notion of endogeny. This specific version of long-range independence turned out to be critical in Aldous [2] for proving the $\zeta$ (2) limit of the random assignment problem. It certainly seems to be an interesting problem to understand better the connection between the endogeny and the version of the long-range independence used in the present paper.

The issue of long-range independence of random combinatorial objects is addressed in a somewhat different, statistical physics context in Mossel [20], Kelly [18], Brightwell and Winkler [11], Rozikov and Suhov [21], and Martin [19], where independent sets (hardcore model) are considered on infinite regular trees, weighted by the Gibbs measure. The long-range interaction between nodes at a large distance is investigated with respect to this measure using the notion of reconstruction. It would be interesting to investigate the connections between the two models.

In a different setting Talagrand [24] proves a certain long-range independence property for the random assignment problem, where the min-weight matching is replaced by the Gibbs distribution on the space of feasible matchings. He uses a rigorous mathematical version of the cavity method, which originated in physics, to prove that the spins (edges of the matching) are asymptotically independent as the size of the problem increases. The particular form of the long-range independence is similar to the one we obtain, refer to Theorem 4 below, and in fact the cavity method, which is based on "knocking" out certain spins from the system and analyzing the relative change of the size of the extremal object, has some similarity with the local weak convergence method, which is also based on considering extremal objects (say independent sets) with one or several nodes excluded. It seems worth investigating whether there is a formal connection between the two methods. Finally, we refer the reader to Hartmann and Weigt [15], who derive the same result as Karp and Sipser for independent sets using nonrigorous arguments from statistical physics.

The rest of the paper is organized as follows. In the following section we describe some prior results on maximum independent sets and matchings in random graphs. Our main theorems are given in Section 3. The operator $T$, fixed point equations, and long-range independence issues are discussed in Section 4. The main results are proven in Section 5. Some conclusions are in Section 6.

We finish this section with some notational conventions. $\operatorname{Exp}(\mu), \operatorname{Pois}(\lambda)$, and $\operatorname{Be}(z)$ denote respectively exponential, Poisson, and Bernoulli distributions with parameters 
$\mu, \lambda>0,0 \leq z \leq 1$. For the Bernoulli distribution $\operatorname{Be}(z)$ we use the convention $\mathbb{P}(X=0)=z, \mathbb{P}(X=1)=1-z$.

\section{PRIOR WORK AND OPEN QUESTIONS}

It is known and simple to prove that $\mathcal{I}(n, r), \mathcal{I}(n, c)=\Theta(n)$ w.h.p. for any all $r \geq 1, c>0$. Moreover, it is known that, w.h.p., $6 \log (3 / 2)-2=.432 \ldots \leq \liminf _{n} \mathcal{I}(n, 3) / n \leq$ $\lim \sup _{n} \mathcal{I}(n, 3) / n \leq .4591$. The lower bound is due to Frieze and Suen [13], and the upper bound is due to Bollobas [10]. The upper bound is generalized for any $r \geq 1$ and uses a very ingenious construction of random regular graphs via matching and random grouping $[9,16]$.

It is natural to expect that the following is true, which unfortunately remains only a conjecture, appearing in several places, most recently in [3] and [5].

Conjecture 1. For every $c>0$ and $r \geq 3$ the limits

$$
\lim _{n \rightarrow \infty} \frac{\mathbb{E}[\mathcal{I}(n, c)]}{n}, \quad \lim _{n \rightarrow \infty} \frac{\mathbb{E}[\mathcal{I}(n, r)]}{n}
$$

exist.

The existence of these limits also implies the convergence to the same limits w.h.p. by applying Azuma's inequality (see [16] for the statement and the applicability of this inequality).

The limit $\lim _{n} \mathbb{E} \mathcal{I}(n, c) / n$ is known to exist for $c \leq e$ and can be computed using the Karp-Sipser [17] algorithm for maximum matching $\mathcal{M}(n, c)$. We describe the algorithm first in high-level terms, and then state the result and its implications to maximum independent sets. The algorithm proceeds in two stages. In the first stage any leaf $v$ in the graph $G(n, c / n)$ is selected. The edge incident to this leaf is selected into a matching, and all the other edges incident to the parent of $v$ are deleted. This is repeated until no leaves are left. In the second stage a matching $M^{*}$ in the remaining graph $G^{*} \subset G$ is selected greedily by selecting at random edges and deleting edges adjacent to the selected one. It is a simple exercise to prove that in the first stage no mistakes are made. That is the matching constructed in the first stage plus the largest matching in the second stage is an optimal matching in the overall graph. Karp and Sipser then show that the matching constructed in the second stage is asymptotically optimal. Using this they prove the following result.

Theorem 1 (Karp and Sipser [17]). For every $c>0$ the maximum matching satisfies

$$
\lim _{n \rightarrow \infty} \frac{\mathbb{E}[\mathcal{M}(n, c)]}{n}=1-\frac{\gamma^{*}(c)+\gamma^{* *}(c)+c \gamma^{*}(c) \gamma^{* *}(c)}{2},
$$

where $\gamma^{*}(c)$ is the smallest solution of the equation $x=\exp (-c \exp (-c x))$ and $\gamma^{* *}(c)=$ $\exp \left(-c \gamma^{*}(c)\right)$. When $c \leq e$, the equation $x=\exp (-c \exp (-c x))$ has a unique solution $\gamma(c)$, and $\left|G^{*}\right|=o(n)$. That is the matching constructed in the first stage is asymptotically optimal as $n \rightarrow \infty$.

The different behavior for $c \leq e$ and $c>e$ is called $e$-cutoff phenomena. Theorem 1 was strengthened later by by Aronson, Pittel, and Frieze [6], who obtain bounds on convergence 
(1). It is the second part of the theorem above that can be used for the analysis of $\mathcal{I}(n, c)$. Observe, that in the first stage of the Karp-Sipser algorithm for every selected leaf if one takes a parent of this leaf instead of an edge between the leaf and its parent, one obtains a node set which is an edge-cover set in the graph $G \backslash G^{*}$ constructed in the first stage. That is every edge in $G \backslash G^{*}$ is incident to at least one node in this cover. It is a simple exercise to prove that this is in fact a minimum node cover in $G \backslash G^{*}$. Its complement is a maximum independent set in $G \backslash G^{*}$. Since $\left|G^{*}\right|=o(n)$ when $c \leq e$, one obtains then the following result.

Corollary 1. When $c \leq e$,

$$
\lim _{n \rightarrow \infty} \frac{\mathbb{E}[\mathcal{I}(n, c)]}{n}=\frac{2 \gamma(c)+c \gamma^{2}(c)}{2} .
$$

The Karp-Sipser algorithm hinges strongly on working with leaves and thus is not applicable to random regular graphs. Moreover, if the edges or the nodes of the graph $G(n, c / n)$ are equipped with weights, then the Karp-Sipser algorithm clearly can produce a strictly suboptimal solution and cannot be used in our setting of weighted nodes and edges. Also, when the edges of $G_{r}(n)$ are equipped with weights, the problem of computing maximum weight matching becomes non-trivial, as opposed to the unweighted case when the full matching exists w.h.p.

\section{MAIN RESULTS}

We begin by introducing the key technique for our analysis-recursive distributional equations and its solutions. This technique was introduced by Aldous [1,2] in the context of solving $\zeta$ (2) limit conjecture for random minimal assignment problem, and was further developed in Aldous and Steele [5], Steele [23], and Aldous and Bandyopadhyay [4, 7]. Let $W$ be a nonnegative random variable with a distribution function $F_{w}(t)=\mathbb{P}(W \leq t)$. We consider four operators $T=T_{\mathcal{I}, r}, T_{\mathcal{I}, c}, T_{\mathcal{M}, r}, T_{\mathcal{M}, c}$ acting on the space of distribution functions $F(t), t \geq 0$, where $c>0$ is a fixed real value and $r \geq 1$ is a fixed integer value.

1. Given $W$ distributed according to $F_{w}$ (we write simply $W \sim F_{w}$ ), and given a distribution function $F=F(t)$, let $B_{1}, B_{2}, \ldots, B_{r} \sim F$ be generated independently. Then $T_{\mathcal{I}, r}: F \mapsto F^{\prime}$, where $F^{\prime}$ is the distribution function of $B^{\prime}$ defined by

$$
B^{\prime}=\max \left(0, W-\sum_{1 \leq i \leq r} B_{i}\right) .
$$

2. Under the same setting as above, let $B_{1}, \ldots, B_{m} \sim F$, where $m$ is a random variable distributed according to Pois $(c)$, independently from $W, B_{i}$. Then $T_{\mathcal{I}, c}: F \mapsto F^{\prime}$, where $F^{\prime}$ is the distribution function of $B^{\prime}$ defined by

$$
B^{\prime}=\max \left(0, W-\sum_{1 \leq i \leq m} B_{i}\right),
$$


when $m \geq 1$ and $B^{\prime}=W$ when $m=0$. For simplicity we identify the sum with zero when $m=0$.

3. Let $W_{1}, \ldots, W_{r} \sim F_{w}, B_{1}, \ldots, B_{r} \sim F$ generated independently. Then $T_{\mathcal{M}, r}: F \mapsto$ $F^{\prime}$, where $F^{\prime}$ is the distribution function of $B^{\prime}$ defined by

$$
B^{\prime}=\max _{1 \leq i \leq r}\left(0, W_{i}-B_{i}\right) .
$$

4. Finally, let $W_{1}, \ldots, W_{m} \sim F_{w}, B_{1}, \ldots, B_{m} \sim F$ generated independently, where $m \sim$ Pois $(c)$, independent from $W_{i}, B_{i}$. Then $T_{\mathcal{M}, c}: F \mapsto F^{\prime}$, where $F^{\prime}$ is the distribution function of $B^{\prime}$ defined by

$$
B^{\prime}=\max _{1 \leq i \leq m}\left(0, W_{i}-B_{i}\right),
$$

when $m \geq 1$ and $B^{\prime}=0$ when $m=0$. Again, for simplicity, we assume that the max expression above is zero when $m=0$.

A distribution function $F$ is defined to be a fixed point distribution of an operator $T$ if $T(F)=F$.

We now state the main result of this paper. Recall, that a distribution function $F(t)$ is defined to be continuous (atom free) if for every $x$ in its support $\lim _{\varepsilon \rightarrow 0}(F(x+\varepsilon)-F(x-\varepsilon))=$ 0 . We use $1\{\cdot\}$ to denote the indicator function.

Theorem 2. Let $F_{w}$ be a continuous nonnegative distribution function. For $r \geq 1$ if the operator $T_{\mathcal{I}, r-1}^{2}$ has a unique fixed point distribution function $F^{*}$, then, w.h.p.,

$$
\lim _{n} \frac{\mathcal{I}_{w}(n, r)}{n}=\mathbb{E}\left[W 1\left\{W-\sum_{1 \leq i \leq r} B_{i}>0\right\}\right],
$$

where $W \sim F_{w}, B_{i} \sim F^{*}$, and $W, B_{i}$ are independent. When $G_{r}(n)$ is replaced by $G(n, c / n)$, the same result holds for $T=T_{\mathcal{I}, c}$, except the sum in the right-hand side of $(7)$ is $\sum_{1 \leq i \leq m} B_{i}$ and $m \sim \operatorname{Pois}(c)$.

Finally, the similar results hold for $\mathcal{M}_{w}(n, r)$ and $\mathcal{M}_{w}(n, c)$ in $G_{r}(n)$ and $G(n, c)$, for $T=T_{\mathcal{M}, r-1}$ and $T=T_{\mathcal{M}, c}$, respectively, whenever the corresponding operator $T$ is such that $T^{2}$ has the unique fixed point distribution $F^{*}$. The corresponding limits are w.h.p.

$$
\lim _{n} \frac{\mathcal{M}_{w}(n, r)}{n}=\frac{1}{2} \mathbb{E}\left[\sum_{1 \leq i \leq r} W_{i} 1\left\{W_{i}-B_{i}=\max _{1 \leq j \leq r}\left(W_{j}-B_{j}\right)>0\right\}\right],
$$

where $W_{i} \sim F_{w}, B_{i} \sim F^{*}$, and

$$
\lim _{n} \frac{\mathcal{M}_{w}(n, c)}{n}=\frac{1}{2} \mathbb{E}\left[\sum_{i \leq m} W_{i} 1\left\{W_{i}-B_{i}=\max _{j \leq m}\left(W_{j}-B_{j}\right)>0\right\}\right],
$$

where $W_{i} \sim F_{w}, B_{i} \sim F^{*}, m \sim \operatorname{Pois}(c)$.

In (9) the value of the indicator function is understood to be zero when $m=0$. For $G=G_{r}(n), G(n, c / n)$ with the weights on nodes given by a distribution function $F_{w}$, let 
$\mathcal{I N}_{w}(n, r), \mathcal{I N}_{w}(n, c)$ denote the cardinality (the number of nodes) of the maximum weight independent set in $G$. In case $F_{w}$ is continuous, the maximum weight independent set is uniquely defined, so $\mathcal{I N}_{w}(n, r), \mathcal{I} \mathcal{N}_{w}(n, c)$ are well defined as well. Clearly, $\mathcal{I}(n, r) \geq$ $\mathcal{I N}_{w}(n, r), \mathcal{I}(n, c) \geq \mathcal{I N}_{w}(n, c) . \mathcal{M N}_{w}(n, r)$ and $\mathcal{M N}_{w}(n, c)$ are defined similarly for matchings. When $T_{\mathcal{I}, r}^{2}, T_{\mathcal{I}, c}^{2}, T_{\mathcal{M}, r}^{2}$, or $T_{\mathcal{M}, c}^{2}$ have the unique fixed point, it is also possible to compute asymptotically $\mathcal{I} \mathcal{N}_{w}(n, r), \mathcal{I N}_{w}(n, c), \mathcal{M N}_{w}(n, r)$, and $\mathcal{M N}_{w}(n, c)$.

Corollary 2. Under the setting of Theorem 2, w.h.p.

$$
\lim _{n} \frac{\mathcal{I N}_{w}(n, r)}{n}=\mathbb{E}\left[1\left\{W-\sum_{1 \leq i \leq r} B_{i}>0\right\}\right],
$$

where $W \sim F_{w}, B_{i} \sim F^{*}, W, B_{i}$ independent, and $F^{*}$ is the unique fixed point distribution of $T^{2}=T_{\mathcal{I}, r-1}^{2}$, and w.h.p.

$$
\lim _{n} \frac{\mathcal{M N}_{w}(n, r)}{n}=\frac{1}{2} \mathbb{E}\left[1\left\{\max _{1 \leq j \leq r}\left(W_{j}-B_{j}\right)>0\right\}\right],
$$

where $W_{i} \sim F_{w}, B_{i} \sim F^{*}, W_{i}, B_{i}$ independent, and $F^{*}$ is the unique fixed point distribution of $T^{2}=T_{\mathcal{M}, r-1}^{2}$ and $W_{i}, B_{i}$ are independent.

Similar results hold for $T=T_{\mathcal{I}, c}, T_{\mathcal{M}, c}$ where again in the sum $\sum_{1 \leq j \leq r}$ we substitute $r$ with random $m \sim \operatorname{Pois}(c)$.

The Theorem 2 is the core result of this paper. It will allow us to obtain several interesting corollaries, which we state below.

Theorem 3. Suppose the weights of the nodes and edges of the graphs $G=G_{r}(n)$ and $G=G(n, c)$ are distributed as $\operatorname{Exp}(1)$. Then:

1. $T_{\mathcal{I}, r-1}^{2}$ has a unique fixed point distribution $F^{*}$ iff $r \leq 4$. In this case, w.h.p.

$$
\lim _{n} \frac{\mathcal{I}_{w}(n, r)}{n}=\frac{(1-b)(r-r b+2 b+2)}{4},
$$

where $b$ is the unique solution of $b=1-\left(\frac{1+b}{2}\right)^{r-1}$. In particular, w.h.p.

$$
\lim _{n} \frac{\mathcal{I}_{w}(n, 2)}{n}=\frac{2}{3}, \quad \lim _{n} \frac{\mathcal{I}_{w}(n, 3)}{n} \approx .6077, \quad \lim _{n} \frac{\mathcal{I}_{w}(n, 4)}{n} \approx .4974
$$

2. $T_{\mathcal{I}, c}^{2}$ has a unique fixed point distribution $F^{*}$ iff $c \leq 2 e$. In this case, w.h.p.

$$
\lim _{n} \frac{\mathcal{I}_{w}(n, c)}{n}=(1-b)\left(1+\frac{c(1-b)}{4}\right)
$$

where $b$ is the unique solution of $1-b=e^{-\frac{c}{2}(1-b)}$. In particular, when $c=2 e$, this limit is $\approx .5517$. 
3. $T_{\mathcal{M}, r-1}^{2}$ has a unique fixed point distribution $F^{*}$ for every $r \geq 1$. Moreover, w.h.p.

$$
\begin{aligned}
\lim _{n} & \frac{\mathcal{M}_{w}(n, r)}{n}=\frac{r b^{r-1}}{2} \int_{0}^{\infty} t e^{-t}\left(1-e^{-t}(1-b)\right)^{r-1} d t \\
& +\frac{r(r-1)(1-b)}{2} \int_{0}^{\infty} \int_{0}^{t} t e^{-t} e^{-z}\left(1-e^{-z}(1-b)\right)^{r-2}\left(1-e^{-t+z}(1-b)\right)^{r-1} d z d t
\end{aligned}
$$

where $b$ is the unique solution of $b=1-\frac{1-b^{r}}{r(1-b)}$.

4. $T_{\mathcal{M}, c}^{2}$ has a unique fixed point distribution $F^{*}$ for all $c>0$. Moreover, for every $c>0$ w.h.p.

$$
\begin{aligned}
\lim _{n} \frac{\mathcal{M}_{w}(n, c)}{n}= & \frac{c}{2} \int_{0}^{\infty} t e^{-t} e^{-c(1-b)\left(1+b-b e^{-t}\right)} d t \\
& +\frac{c^{2}}{2} \int_{0}^{\infty} \int_{0}^{t} t e^{-t} e^{-z}\left(1-e^{-t+z}(1-b)\right) \\
& \quad \times e^{-c+c\left(1-e^{-t+z}(1-b)\right)\left(1-e^{-z}(1-b)\right)} d z d t
\end{aligned}
$$

where $b$ is the unique solution of $1-e^{-c b}=c(1-b)^{2}$.

The result above generalizes to the case when $\operatorname{Exp}(1)$ is replaced by $\operatorname{Exp}(\mu)$ for any $\mu>0$, since, for any $\alpha>0$ and $W \sim \operatorname{Exp}(1), \alpha W \sim \operatorname{Exp}(1 / \alpha)$. The expression in (15) involving integrals is similar to the one found in [5] for maximum weight matching on a tree. It is a pleasant surprise, though, that the answers for independent sets are derived in closed form.

Part 2 of Theorem 3 leads to an interesting phase transition behavior. For $F_{w}=\operatorname{Exp}(1)$ our result says that the value $c=2 e$ is a phase transition point for the operator $T_{\mathcal{I}, c}^{2}$ where for $c \leq 2 e$ the operator has a unique fixed point distribution, but for $c>2 e$ the fixed point distribution is not unique. Contrast this with $e$-cutoff phenomena described above. It turns out (see Theorems 8 and 9 below) that this phase transition is directly related to some long-range independence/dependence property of the maximum weight independent sets in the underlying graph $G(n, c / n)$. Curiously, no such phase transition occurs for maximum weight matchings.

As a sanity check, let us show directly the validity of (12) for the case $r=1$. The answer given by the formula is $3 / 4$. It is, though, easy to compute $\mathcal{I}_{w}(n, 1)$ exactly. The graph is a collection of $n / 2$ isolated edges. For each edge we simply select the the incident node with larger weight, which by memoryless property of Exp is $1 / 2+1=3 / 2$. The limit $\mathcal{I}_{w}(n, 1) / n \rightarrow 3 / 4$ then checks.

The formula in (15), although it involves a double integration, is in fact simple to compute explicitly. After expanding the expressions involving powers $r-1$ and $r-2$ and integrating with respect to $z$, the resulting expression is a combination of the terms of the form $t^{l} e^{-k t}$ for some integers $l, k$ which need to be integrated with respect to $t$ and this can be done using the integration by parts. We demonstrate this for the case $r=2$, but we do not compute the double integral explicitly as the resulting expression is not very revealing. Before we do the computations for the case $r=2$, we note that we expect the answer to be $2 / 3$. The reason is that the random 2-regular graph is a collection of disjoint cycles on $n$ elements. It is well 
known that, w.h.p., these cycles have decreasing lengths, starting from $\Theta\left(n^{\frac{1}{2}}\right)$. Clearly on each even cycle the largest weighted matching is equal to the largest weighted independent set. On odd cycles they differ by at most a constant and since most of the cycles in random 2-regular graphs are "large" this difference is negligible. On the other hand, we know from (13) that the limiting constant for the largest weighted independent set in 2-regular random graph is $(2 / 3)$. We now demonstrate that this intuition is indeed correct and the largest weighted matching is also equal to $2 / 3$. The expression (15) corresponding to $r=2$ is

$$
\begin{aligned}
\frac{2 b}{2} \int_{0}^{\infty} t e^{-t} & \left(1-e^{-t}(1-b)\right) d t+\frac{2(1-b)}{2} \int_{0}^{\infty} \int_{0}^{t} t e^{-t} e^{-z}\left(1-e^{-t+z}(1-b)\right) d z d t \\
& =b \int_{0}^{\infty}\left(t e^{-t}-t e^{-2 t}(1-b)\right) d t+(1-b) \int_{0}^{\infty} t e^{-t} \int_{0}^{t}\left(e^{-z}-e^{-t}(1-b)\right) d z d t \\
& =b\left(1-\frac{1-b}{4}\right)+(1-b) \int_{0}^{\infty} t e^{-t}\left(1-e^{-t}-t e^{-t}(1-b)\right) d t \\
& =b\left(1-\frac{1-b}{4}\right)+(1-b)\left(1-\frac{1}{4}-\frac{1-b}{4}\right) \\
& =\frac{b+1}{2}
\end{aligned}
$$

The value for $b$ is easily computed to be $1 / 3$ from the corresponding equation. This results in the value $2 / 3$ which matches (13).

Theorems 2 and 3 also hold for a simpler model of a 2-regular graph $-n$-cycle. The $n$ nodes $0,1, \ldots, n-1$ and edges $(0,1), \ldots,(n-2, n-1),(n-1,0)$ of this cycle are assumed to have weights distributed according to some distribution function $F_{w}$. Let $I_{w}(n$, cycle) and $M_{w}(n$, cycle) denote respectively the maximum weight of an independent set and the maximum weight of a matching.

Corollary 3. Suppose $T_{\mathcal{I}, 2}^{2}$ has a unique fixed point. Then (7), (8) hold when $r=2$ and $\mathcal{I}_{w}\left(n\right.$, cycle) replaces $\mathcal{I}_{w}(n, 2)$ and $\mathcal{M}_{w}\left(n\right.$,cycle) replaces $\mathcal{M}_{w}(n, 2)$. When $F_{w}=\operatorname{Exp}(1)$, w.h.p.

$$
\lim _{n} \frac{\mathcal{I}_{w}(n, \text { cycle })}{n}=\lim _{n} \frac{\mathcal{M}_{w}(n, \text { cycle })}{n}=\frac{2}{3}
$$

We can also compute the cardinality of independent sets which achieve the maximum weight, when $F_{w}=\operatorname{Exp}(1)$.

Corollary 4. When $F_{w}=\operatorname{Exp}(1)$, w.h.p.

$$
\begin{aligned}
\liminf _{n} \frac{\mathcal{I}(n, 2)}{n} \geq \lim _{n} \frac{\mathcal{I} \mathcal{N}_{w}(n, 2)}{n}=\frac{4}{9}, \liminf _{n} \frac{\mathcal{I}(n, 3)}{n} \geq \lim _{n} \frac{\mathcal{I} \mathcal{N}_{w}(n, 3)}{n} \approx .3923, \\
\liminf _{n} \frac{\mathcal{I}(n, 4)}{n} \geq \lim _{n} \frac{\mathcal{I} \mathcal{N}_{w}(n, 4)}{n} \approx .3533 .
\end{aligned}
$$


We note that for $r=3,4$ our lower bounds $.3923 n-o(n), .3533 n-o(n)$ are, unfortunately, weaker than then the state of the art bounds $.4328 n-o(n)$ and $.3901 n-o(n)$ established in [13] and [25].

An important implication of the uniqueness of the fixed point distribution of $T^{2}$ for the types of $T$ described above, is that it implies a certain long-range independence properties of the structures we consider. The following theorem makes this notion precise. While the theorem is not used directly in this paper, we believe it is interesting by itself. Below $G$ denotes one of the graphs $G_{r}(n)$ or $G(n, c)$. Let $E$ denote the (random) edge set of $G$.

Theorem 4. Let $T$ be one of the four operators (3),(4),(5),(6) with respect to some continuous distribution function $F_{w}$, and let $\mathcal{C}_{w}(n)=\mathcal{I}_{w}(n, r), \mathcal{I}_{w}(n, c), \mathcal{M}_{w}(n, r)$, or $\mathcal{M}_{w}(n, c)$. Denote by $\mathcal{O}(n)$ the subset of $[n]$ or $E$ (depending on a context), which achieves $\mathcal{C}_{w}(n)$. Select two elements $i, j$ of $[n]$ or E uniformly at random. If $T^{2}$ has a unique fixed point distribution, then

$$
\mathbb{P}(i, j \in \mathcal{O}(n)) \rightarrow \mathbb{P}(i \in \mathcal{O}(n)) \mathbb{P}(j \in \mathcal{O}(n)),
$$

as $n \rightarrow \infty$.

Recall that for each of the four objects $|\mathcal{C}(n)|=\Theta(n)$ w.h.p. As a result the values $\mathbb{P}(v \in \mathcal{O}(n))$ do not vanish and the theorem above does have a nontrivial content (the limit is not zero). In fact we will show a much stronger result stating that, when $T^{2}$ has a unique fixed point, the event $v \in \mathcal{O}(n)$ is almost independent of the portion of the graph $G$ outside a depth- $d$ graph-theoretic neighborhood of $v$, when $d$ is a sufficiently large constant integer.

\section{FIXED POINTS OF THE OPERATOR $T^{2}$ AND THE LONG-RANGE INDEPENDENCE}

\subsection{Maximum Weight Independent Sets and Matchings in Trees. Fixed Points of $T^{2}$ and the Bonus Function}

We start by analyzing operator $T$-any of the four operators introduced in the previous section. Given two distribution functions $F_{1}, F_{2}$ defined on $[0, \infty)$, we say that $F_{2}$ stochastically dominates $F_{1}$ and write $F_{1} \prec F_{2}$ if $F_{1}(t) \geq F_{2}(t)$ for every $t \geq 0$. A sequence of distribution functions $F_{n}$ is defined to converge weakly to a distribution function $F$ (written $\left.F_{n} \Rightarrow F\right)$ if $\lim _{n} F_{n}(t)=F(t)$ for every $t$ which is a point of continuity of $F$.

Lemma 5. The operators $T=T_{\mathcal{I}, r}, T_{\mathcal{I}, c}, T_{\mathcal{M}, r}, T_{\mathcal{I}, c}$ are continuous with respect to the weak convergence. That is, given a sequence of distributions $F, F_{s}, s=1,2, \ldots$, if $F_{s} \Rightarrow F$, then $T\left(F_{s}\right) \Rightarrow T(F)$.

Proof. The proof is almost immediate for $T=T_{\mathcal{I}, r}$. Since the summation, subtraction, and max operations are continuous functions then the assertion holds. Here we use the fact that for any continuous function $f, F_{n} \Rightarrow F$ implies $f\left(F_{n}\right) \Rightarrow f(F)$ (see Continuous Mapping Theorem in [12]). The proof for the case $T=T_{\mathcal{I}, c}$ is slightly more subtle since we are dealing with sum of randomly many elements. Let $X_{1}^{(n)}, X_{2}^{(n)}, \ldots$ be i.i.d. sample from the distribution $F_{n}$, and let $m$ be a Poisson $(c)$ random variable which is independent of $\left(X_{j}^{(n)}\right)_{j \geq 1}$. 
Let also $X_{1}, X_{2}, \ldots$, be an i.i.d. sample from $F$. Define $X^{(n)}=\max \left(0, W-\sum_{1<i<m} X_{i}^{(n)}\right)$ and $X=\max \left(0, W-\sum_{1<i<m} X_{i}\right)$. Fix arbitrary $\varepsilon>0$ and $m_{0}$ such that $m$ does not exceed $m_{0}$ with probability at least $1-\varepsilon$. For any fixed $t \geq 0$ and $n$

$$
\left|\mathbb{P}\left(X^{(n)} \leq t\right)-\mathbb{P}\left(X^{(n)} \leq t \mid m \leq m_{0}\right) \mathbb{P}\left(m \leq m_{0}\right)\right| \leq \varepsilon,
$$

and

$$
\left|\mathbb{P}(X \leq t)-\mathbb{P}\left(X \leq t \mid m \leq m_{0}\right) \mathbb{P}\left(m \leq m_{0}\right)\right| \leq \varepsilon,
$$

Now, we have $\left|\mathbb{P}\left(X^{(n)} \leq t \mid m \leq m_{0}\right)-\mathbb{P}\left(X \leq t \mid m \leq m_{0}\right)\right| \leq \varepsilon$ for sufficiently large $n$ since $F_{n} \Rightarrow F$ and $m$ is conditioned to be at most $m_{0}$. Combining, we obtain $\mid \mathbb{P}\left(X^{(n)} \leq t\right)-\mathbb{P}(X \leq$ $t) \mid \leq 3 \varepsilon$ for sufficiently large $n$. This completes the proof for $T=T_{\mathcal{I}, c}$. The proofs for $T=T_{\mathcal{M}, r}, T=T_{\mathcal{M}, c}$ are similar.

Let 0 denote (for simplicity) the distribution function of a random variable $X$ which is zero w.p.1. Let $W_{r}^{\max }=\max _{1 \leq i \leq r} W_{i}$, where $W_{i} \sim F_{w}$ are independent. Let also $W_{c}^{\max }=$ $\max _{1 \leq i \leq m} W_{i}$, where $W_{i} \sim F_{w}$ are independent and $m \sim \operatorname{Pois}(c)$. Denote by $F_{w, r}$ and $F_{w, c}$ the distribution functions of $W_{r}^{\max }$ and $W_{c}^{\max }$, respectively.

Proposition 1. Fix $r \geq 1, c>0$, a distribution function $F_{w}(t), t \geq 0$ and $T=T_{\mathcal{I}, r}$ or $T_{\mathcal{I}, c}$. As $s \rightarrow \infty$, the two sequences of distributions $T^{2 s}(0), T^{2 s}\left(F_{w}\right)$ weakly converge to distribution functions $F_{* *}, F^{* *}$, respectively, which are fixed points of the operator $T^{2}$. For any distribution function $F_{0}$ with nonnegative support, $T^{2 s}(0) \prec T^{2 s+2}\left(F_{0}\right) \prec T^{2 s}\left(F_{w}\right)$ and $T^{2 s+1}\left(F_{w}\right) \prec T^{2 s+3}\left(F_{0}\right) \prec T^{2 s+1}(0)$ for all $s=1,2, \ldots$.

If the operator $T$ is such that $T^{2}$ has a unique fixed point (and $F_{* *}=F^{* *} \equiv F^{*}$ ), then for any distribution function $F_{0}$ with nonnegative support, $T^{s}\left(F_{0}\right) \Rightarrow F^{*}$ as $s \rightarrow \infty$. In particular, $T^{s}(0), T^{s}\left(F_{w}\right) \rightarrow F^{*}$. Moreover, $F^{*}$ is also the unique fixed point of $T$.

When $T=T_{\mathcal{M}, r}$ or $T=T_{\mathcal{M}, c}$, the same result holds with $F_{w, r}$ and $F_{w, c}$ respectively replacing $F_{w}$.

Proof. Let $F=F(t), t \geq 0$ be a distribution function corresponding to some nonnegative random variable. It follows immediately from the definitions that

$$
T_{\mathcal{I}, r}(F) \prec F_{w}, \quad T_{\mathcal{I}, c}(F) \prec F_{w}, \quad T_{\mathcal{M}, r}(F) \prec F_{w, r}, \quad T_{\mathcal{M}, c}(F) \prec F_{w, c} .
$$

Observe that each of the four operators $T$ above is antimonotone. That is, if $F_{1} \prec F_{2}$ for some distribution functions $F_{1}, F_{2}$, then $T\left(F_{1}\right) \succ T\left(F_{2}\right)$. Applying this twice, we obtain $T^{2}\left(F_{1}\right) \prec T^{2}\left(F_{2}\right)$. Trivially, $0 \prec T^{2}(0)$. Then $0 \prec T^{2}(0) \prec T^{4}(0) \prec \cdots$, and this sequence weakly converges to some function $F_{* *}=F_{* *}(t) \leq 1$, since the values of $T^{2 s}(0)$ at each fixed $t$ are decreasing and are bounded below by 0 . Note that $F_{* *}(t)$ is a nondecreasing function of $t$, since this is the case for each distribution $T^{2 s}(0)$. Finally, from (21) we have that $\lim _{t \rightarrow \infty} F_{* *}(t)=1$. Thus $F_{* *}(t)$ is a distribution function. As $T^{2 s}(0) \Rightarrow F_{* *}$ and by Lemma $5, T$ is continuous, we have $T^{2}\left(F_{* *}\right)=F_{* *}$.

We now fix $T=T_{\mathcal{I}, r}$. The proof of the other three cases is very similar. From (21), by taking $F=T\left(F_{w}\right)$, we obtain $T^{2}\left(F_{w}\right) \prec F_{w}$. Then, by monotonicity of $T^{2}$ we obtain $F_{w} \succ T^{2}\left(F_{w}\right) \succ \cdots \succ T^{2 s}\left(F_{w}\right)$ and the sequence $T^{2 s}\left(F_{w}\right)$ converges weakly to some function $F^{* *}$. We repeat the arguments above to show that $F^{* *}$ is actually a distribution function. Again applying Lemma 5, we conclude $T^{2}\left(F^{* *}\right)=F^{* *}$. 
For any distribution function $F_{0}$ with nonnegative support we have $0 \prec T\left(F_{0}\right), T^{2}\left(F_{0}\right) \prec$ $F_{w}$, where again we use nonnegativity and $(21)$, first applied to $F_{0}$ and then to $T\left(F_{0}\right)$. Applying the monotonicity

$$
T^{2 s}(0) \prec T^{2 s+1}\left(F_{0}\right), T^{2 s+2}\left(F_{0}\right) \prec T^{2 s}\left(F_{w}\right),
$$

and therefore $T^{2 s+1}(0) \succ T^{2 s+3}\left(F_{0}\right) \succ T^{2 s+1}\left(F_{w}\right)$. This completes the proof of the first part of the proposition.

Suppose now $T^{2}$ has a unique fixed point $F^{*}=F^{* *}=F_{* *}$. For any distribution function $F_{0}$ we have $0 \prec T\left(F_{0}\right), T^{2}\left(F_{0}\right) \prec F_{w}$, where again we use (21). Applying (22) and using $T^{2 s}(0), T^{2 s}\left(F_{w}\right) \Rightarrow F^{*}$, we obtain $T^{s}\left(F_{0}\right) \Rightarrow F^{*}$. In particular, by taking $F_{0}=0$ and $F_{0}=F_{w}$, we obtain $T^{s}(0), T^{s}\left(F_{w}\right) \Rightarrow F^{*}$. Finally, we obtain $T^{2 s}\left(T\left(F^{*}\right)\right) \Rightarrow F^{*}$. But $T^{2 s}\left(T\left(F^{*}\right)\right)=T\left(T^{2 s}\left(F^{*}\right)\right)=T\left(F^{*}\right)$. Thus $T\left(F^{*}\right)=F^{*}$. Clearly it is the unique fixed point of $T$ since any fixed point of $T$ is also a fixed point of $T^{2}$.

We now switch to analyzing the maximum weight independent set problem on a tree. The derivation here repeats the development in [5] for maximum weight matching in random trees. We highlight important differences where appropriate.

Suppose we have a (nonrandom) finite tree $H$ with nodes $0,1, \ldots, h=|H|-1$, with a fixed root 0 . The nodes of this tree are equipped with some (nonrandom) weights $W_{0}, W_{1}, \ldots, W_{h} \geq 0$. For any node $i \in H$, let $H(i)$ denote the subtree rooted at $i$ consisting of all the descendants of $i$. In particular, $H(0)=H$. Let $\mathcal{I}_{H(i)}$ denote the maximum weight of an independent set in $H(i)$ and let $B_{H(i)}=\mathcal{I}_{H(i)}-\sum_{j} \mathcal{I}_{H(j)}$, where the sum runs over nodes $j$ which are children of $i$. If $i$ has no children, then $B_{H(i)}$ is simply $\mathcal{I}_{H(i)}=W_{i}$. Observe that $B_{H(i)}$ is also a difference between $\mathcal{I}_{H(i)}$ and the maximum weight of an independent set in $H(i)$, which is not allowed to use node $i$. Clearly, $0 \leq B_{H(i)} \leq W_{i}$. The value $B_{H(i)}$ was considered in [5] in the context of maximum weight matchings and was referred to as a bonus of a node $i$ in tree $H(i)$. W.l.g. denote by $1, \ldots, m$ the children of the root node 0 .

\section{Lemma 6.}

$$
B_{H}=\max \left(0, W_{0}-\sum_{1 \leq i \leq m} B_{H(i)}\right) .
$$

Moreover, if $W_{0}>\sum_{1 \leq i \leq m} B_{H(i)}$ (that is, if $B_{H(0)}>0$ ), then the maximum weight independent set in $H$ must contain node 0 . If $W_{0}<\sum_{1 \leq i \leq m} B_{H(i)}$, then the maximum weight independent set in $H$ does not contain the node 0.

Remark. There might be several independent sets in $H$ which achieve $\mathcal{I}_{H}$. The second part of the lemma refers to any independent set achieving maximum weight. Also, the statement of the lemma applies as well to every node $i$ with respect to its tree $H(i)$. That is let $j_{1}, \ldots, j_{l}$ be the children of a node $i$. Then the lemma claims $B_{H(i)}=\max \left(0, W_{0}-\sum_{1 \leq i \leq l} B_{H\left(j_{i}\right)}\right)$.

Proof. Consider an independent set $V \subset H$ which achieves the maximum weight. We take an arbitrary such in case there are many. If $0 \in V$, then $i \notin V$ for $1 \leq i \leq m$. Then $V$ is obtained by taking maximum independent sets $V_{i}$ in $H(i)$ such that $i \notin V(i)$. By definition 
the weight of such $V_{i}$ is $\mathcal{I}_{H(i)}-B_{H(i)}$. On the other hand, if $0 \notin V$, then a maximum weight of an independent set in $H$ is obtained simply as $\sum_{1 \leq i \leq m} \mathcal{I}_{H(i)}$. We conclude that

$$
\mathcal{I}_{H}=\max \left(W_{0}+\sum_{1 \leq i \leq m}\left(\mathcal{I}_{H(i)}-B_{H(i)}\right), \sum_{1 \leq i \leq m} \mathcal{I}_{H(i)}\right) .
$$

Recall that $B_{H}=\mathcal{I}_{H}-\sum_{1 \leq i \leq m} \mathcal{I}_{H(i)}$. Subtracting $\sum_{1 \leq i \leq m} \mathcal{I}_{H(i)}$ from both sides of (24) we obtain (23). The second part of the lemma follows directly from the discussion above.

A similar development is possible for maximum weight matching. Suppose the edges of the tree $H$ are equipped with weights $W_{i, j}$. Let $\mathcal{M}_{H(i)}$ denote the maximum weight of a matching in $H(i)$, and let $B_{H(i)}$ denote the difference between $\mathcal{M}_{H(i)}$ and the maximum weight of a matching in $H(i)$, which is not allowed to include any edge in $H(i)$ incident to $i$. Again $1,2, \ldots, m$ are assumed to be the children of the root 0 .

\section{Lemma 7.}

$$
B_{H}=\max \left(0, \max _{1 \leq i \leq m}\left(W_{0, i}-B_{H(i)}\right)\right) .
$$

Moreover, if $W_{0, i}-B_{H(i)}>W_{0, i^{\prime}}-B_{H\left(i^{\prime}\right)}$ for all $i^{\prime} \neq i$ and $W_{0, i}-B_{H(i)}>0$, then every maximum weight matching in $H$ contains edge $(0, i)$. If $W_{0, i}-B_{H(i)}<0$ for all $i=1, \ldots, m$, then every maximum weight matching in $H$ does not contain any edge incident to 0.

Proof. The proof is very similar to the one of Lemma 6. If a maximum weight matching contains edge $(0, i)$, then its weight is $W_{0, i}$ plus maximum weight of a matching in $H(i)$ with node $i$ excluded, plus the sum of the maximum weights of matchings in $H(j), 1 \leq j \leq$ $m, j \neq i$. If a maximum weight matching contains none of the edges $(0, i), 1 \leq i \leq m$, then its weight is simply sum of maximum weight of matchings in $H(i)$ for all $i=1, \ldots, m$. We obtain

$$
\mathcal{M}_{H}=\max \left(\max _{1 \leq i \leq m}\left(W_{0, i}+\mathcal{M}_{H(i)}-B_{H(i)}+\sum_{j \neq i} \mathcal{M}_{H(j)}\right), \sum_{1 \leq i \leq m} \mathcal{M}_{H(j)}\right) .
$$

Subtracting $\sum_{1 \leq j \leq m} \mathcal{M}_{H(j)}$ from both sides of (26), we obtain (25). The proof of the second part follows immediately from the discussion above.

\subsection{Long-Range Independence}

We now consider trees $H$ of specific types. Given integers $r \geq 2, d \geq 2$, let $H_{r}(d)$ denote an $r$-regular finite tree with depth $d$. The root node 0 has degree $r-1$, all the nodes at distance $\geq 1, \leq d-1$ from the root have outdegree $r-1$, and all the nodes at distance $d$ from 0 are leaves. (Usually, in the definition of an $r$-regular tree, the root node is assumed to have degree $r$, not $r-1$. The slight distinction here is done for convenience.) Also, given a constant $c>0$, a Poisson tree $H(c, d)$ with parameter $c$ and depth $d$ is constructed as follows. The root node has a degree which is a random variable distributed according to Pois $(c)$ distribution. All the children of 0 have outdegrees which are also random, distributed according to Pois $(c)$. In particular, the children of 0 have total degrees $1+$ Pois $(c)$. Similarly, 
children of children of 0 also have outdegree Pois $(c)$, etc. We continue this process until either the process stops at some depth $d^{\prime}<d$, where no nodes in level $d^{\prime}$ have any children, or until we reach level $d$. In this case all the children of the nodes in level $d$ are deleted and the nodes in level $d$ become leaves. We obtain a tree with depth $\leq d$. We call this a depth- $d$ Poisson tree.

Let $H=H_{r}(d)$ or $H(c, d)$. Suppose the nodes and the edges of $H$ are equipped with weights $W_{i}, W_{i, j}$, which are generated at random independently using a distribution function $F_{w}$. Fix any infinite sequences $\bar{w}=\left(w_{1}, w_{2}, \ldots\right) \in[0, \infty)^{\infty}$ and $\bar{b}=\left(b_{1}, b_{2}, \ldots\right) \in\{0,1\}^{\infty}$. For every $i=1,2, \ldots, d$ let $i 1, i 2, \ldots, i j_{i}$ denote the nodes of $H$ in level $i$ (if any exist for $H(c, d))$. When $H=H_{r}(d), j_{i}=(r-1)^{i}$, of course. Let $(\mathcal{I} \mid(\bar{b}, \bar{w}))$ denote the maximum weight of an independent set $V$ in $H$ such that the nodes $d j$ with $b_{j}=1$ are conditioned to be in $V$, nodes $d j$ with $b_{j}=0$ are conditioned not to be in $V$, and the weights of nodes $d j$ are conditioned to be equal to $w_{j}$ for $j=1, \ldots, j_{d}$. That is, we consider the maximum weight of independent set among those which contain depth $d$ leaves with $b_{j}=1$, do not contain depth $d$ leaves with $b_{j}=0$, and with the weights of the leaves deterministically determined by $\bar{w}$. For brevity we call it the maximum weight of an independent set with boundary condition $(\bar{b}, \bar{w})$. For the case $H=H(c, d)$, the boundary condition is simply absent when the tree does not contain any nodes in the last level $d .\left(\mathcal{I}_{H(i j)} \mid(\bar{b}, \bar{w})\right)$ are defined similarly for the subtrees $H(i j)$ spanned by nodes $i j$ in level $i$. Given again $\bar{b}$, $\bar{w}$, let $(\mathcal{M} \mid(\bar{b}, \bar{w}))$ and $\left(\mathcal{M}_{H(i j)} \mid(\bar{b}, \bar{w})\right)$ denote, respectively, the maximum weight of a matching $E$ in $H$ and $H_{i j}$, such that the edges incident to nodes $d j$ are conditioned to be in $E$ when $b_{j}=1$, edges incident to nodes $d j$ are conditioned not to be in $E$ when $b_{j}=0$, and the weights of the edges incident to nodes $d j$ are conditioned to be equal to $w_{j}, j=1, \ldots, j_{d}$ (of course, we refer to edges between nodes in levels $d-1$ and $d$ as there is only one edge per each leaf in level $d$ ).

For the case of independent sets, let $(B \mid(\bar{b}, \bar{w}))$ denote the bonus of the root node 0 given the boundary condition $(\bar{b}, \bar{w})$. Namely,

$$
(B \mid(\bar{b}, \bar{w}))=(\mathcal{I} \mid(\bar{b}, \bar{w}))-\sum_{1 \leq j \leq j_{1}}\left(\mathcal{I}_{H(1 j)} \mid(\bar{b}, \bar{w})\right) .
$$

For the case of matchings, let $(B \mid(\bar{b}, \bar{w}))$ also denote the bonus of the root node 0 given the boundary condition $(\bar{b}, \bar{w})$. Namely,

$$
(B \mid(\bar{b}, \bar{w}))=(\mathcal{M} \mid(\bar{b}, \bar{w}))-\sum_{1 \leq j \leq j_{1}}\left(\mathcal{M}_{H(1 j)} \mid(\bar{b}, \bar{w})\right) .
$$

It should be always clear from the context whether $B$ is taken with respect to independent sets or matchings.

The following theorem establishes the crucial long-range independence property for the maximum weight independent sets and matchings in trees $H=H_{r}(d), H(c, d)$ when the corresponding operator $T^{2}$ has a unique fixed point. It establishes that the distribution of the bonus $(B \mid(\bar{b}, \bar{w}))$ of the root is asymptotically independent of the boundary condition $(\bar{b}, \bar{w})$ as $d$ becomes large. Recall our convention that 0 denotes the distribution of a random variable which is equal to zero with probability one.

Theorem 8. Given a distribution function $F_{w}$ and a regular tree $H=H_{r}(d)$ let $T=T_{\mathcal{I}, r}$. Then for every $t \geq 0$ and $\bar{b}, \bar{w}$

$$
T^{d-1}(0)(t) \leq \mathbb{P}((B \mid(\bar{b}, \bar{w})) \leq t) \leq T^{d-1}\left(F_{w}\right)(t)
$$


when $d$ even, and

$$
T^{d-1}\left(F_{w}\right)(t) \leq \mathbb{P}((B \mid(\bar{b}, \bar{w})) \leq t) \leq T^{d-1}(0)(t)
$$

when $d$ is odd. Suppose in addition the operator $T^{2}=T_{\mathcal{I}, r-1}^{2}$ has the unique fixed distribution $F^{*}$. Then for every $t \geq 0$

$$
\sup _{\bar{b}, \bar{w}}\left|\mathbb{P}((B \mid(\bar{b}, \bar{w})) \leq t)-F^{*}(t)\right| \rightarrow 0
$$

as $d \rightarrow \infty$. Similar assertion holds for $T=T_{\mathcal{M}, r}$ and for $H=H(c, d)$ with $T=T_{\mathcal{I}, c}$ and $T=T_{\mathcal{M}, c}$. For the cases $T=T_{\mathcal{M}, r}$ and $T_{\mathcal{M}, c}, F_{w}$ is replaced with $F_{w, r}$ and $F_{w, c}$ respectively.

Before we prove the proposition above, which is one of the key results of the paper, let us compare it with the developments in [5]. In that paper maximum weight matching is considered on an $n$-node tree, drawn independently and uniformly from the space of all $n^{n-2}$ labelled trees. The notion of a bonus is introduced and the recursion (25) is derived. However, since a tree structure is assumed to begin with, there is no need to consider the boundary conditions $(\bar{b}, \bar{w})$. Here we avoid the difficulty of the nontree structure by proving the long-range independence property via the uniqueness of fixed points of $T^{2}$.

Proof. We prove (28) and (27) for independent sets in $H=H_{r}(d)$. The proof of (29) will then be almost immediate. The proofs for other cases are similar. We will indicate the differences where appropriate. The proof of (28) and (27) proceeds by induction in $d$. Suppose $d=1$. Then we have

$$
(B \mid(\bar{b}, \bar{w}))=\max \left(0, W_{0}-\sum_{i: 1 \leq i \leq r-1, \bar{b}_{1 i}=1} \bar{w}_{1 i}\right) \leq W_{0} .
$$

Then we obtain the bound (28) for the case $d=1$. Suppose the assumption holds for $d-1$ and $d$ is odd. We have

$$
(B \mid(\bar{b}, \bar{w}))=\max \left(0, W_{0}-\sum_{i: 1 \leq i \leq r-1}\left(B_{1 i} \mid(\bar{b}, \bar{w})\right)\right) .
$$

By the inductive assumption the distribution of each of $\left(B_{1 i} \mid(\bar{b}, \bar{w})\right)$ dominates $T^{d-2}(0)$ and is dominated by $T^{d-2}\left(F_{w}\right)$. Applying this to (30), we obtain (28). The case $d$ is even is considered similarly. This completes the induction and establishes (28) and (27). Now if $T^{2}$ has the unique fixed point distribution $F^{*}$, then, by Proposition $1, T^{d}(0), T^{d}\left(F_{w}\right)$ converge to $F^{*}$ as $d \rightarrow \infty$, and we obtain (29). The proof for the case of Poisson tree $H(c, d)$ instead of $H_{r}(d)$ is pretty much identical. For the proof of the same result for maximum weight matching we just use $F_{w, r}$ and $F_{w, c}$ instead of $F_{w}$, just as we did in the proof of Proposition 1.

While not used for the further results in this paper, it is interesting to note that the uniqueness of the solution $T^{2}(F)=F$ is the tight condition for (29), as the following theorem indicates. 
Theorem 9. Under the setting of Theorem 9 suppose the operator $T^{2}$ has more than one fixed point distributions $F^{*}$. Then for every such $F^{*}$ there exists $t \geq 0$ such that

$$
\limsup _{d} \sup _{\bar{b}, \bar{w}}\left|\mathbb{P}((B \mid(\bar{b}, \bar{w})) \leq t)-F^{*}(t)\right|>0 .
$$

Proof. As usual we start with $T=T_{\mathcal{I}, r}$. The proofs for other cases are similar, we highlight the differences where appropriate. Let $F_{* *}$ and $F^{* *}$ be distributions introduced in Proposition 1 . The nonuniqueness of the fixed point of $T^{2}$ implies that $F_{* *} \neq F^{* *}$. For every node $j$ in layer $d$ (last layer) of $H_{r}(d)$ set $b_{j}=0, w_{j}=0$. In particular, the bonus $B_{j}$ of each such node is zero. Then for every node in layer $d-1$ its bonus is given by the distribution $T(0)=F_{w}$, the bonus of each node in layer $d-2$ has distribution $T^{2}(0)$, etc. The root node 0 has bonus with distribution $T^{d}(0)$. When $d$ is an even number diverging to infinity, from Proposition $1, T^{d}(0)$ converges weakly to $F_{* *}$. Thus the distribution of $(B \mid(\bar{b}, \bar{w}))$ converges to $F_{* *}$. If $F^{*}$, a fixed point of $T^{2}$, is distinct from $F_{* *}$, then we obtain that (31) holds. Suppose, on the other hand, $F^{*}=F_{* *}$. We claim that $T\left(F_{* *}\right) \neq F_{* *}$. Assuming this is the case, we consider the same boundary condition $\bar{b}=\bar{w}=0$ but take $d$ to be odd integer diverging to infinity. Then the bonus of the root 0 converges in distribution to $T\left(F_{* *}\right) \neq F_{* *}$ and (31) is shown again.

Assume $T\left(F_{* *}\right)=F_{* *}$. Recall from the first part of Proposition 1 that for every distribution $F_{0}, T^{2 s+3}\left(F_{0}\right) \prec T^{2 s+1}(0)$. Taking $F_{0}=T\left(F^{* *}\right)$, we obtain $F^{* *}=T^{2 s+4}\left(F^{* *}\right) \prec$ $T^{2 s+1}(0)$. Taking $s \rightarrow \infty, F^{* *} \prec T\left(F_{* *}\right)=F_{* *}$. But, from Proposition $1, F_{* *} \prec F^{* *}$, and, as a result, $F_{* *}=F^{* *}$, implying (again using Proposition 1) $T^{2}$ has the unique fixed point. We obtained a contradiction.

\section{APPLICATIONS TO MAXIMUM WEIGHT INDEPENDENT SETS AND MATCHINGS IN $G_{r}(n)$ AND $G(n, c / n)$}

\subsection{Long-Range Independence in $G_{r}(n), G(n, c / n)$}

The goal of the current section is to demonstrate that Theorem 8 allows us to reduce the computation of the maximum weight independent set and the maximum weight matching in random graphs to a much simpler problem of finding those in trees. We highlight this key message of the paper as the following local optimality property: If the operator $T^{2}$ corresponding to a maximum weight combinatorial object (independent set or matching) in a sparse random graph has a unique fixed point, then, for a randomly selected node (edge) of the graph, the event "the node (edge) belongs to the optimal object" and the distribution of the node (edge) weight, conditioned that it does, asymptotically depends only on the constant size neighborhood of the node and is independent from the rest of the graph. In other words, when $T^{2}$ has a unique fixed point, the maximum weight independent sets and matchings exhibit a long-range independence property.

Our hope is that similar local optimality can be established for other random combinatorial structures, for example, the problem of coloring in sparse random graphs and also the problems of Gibbs distributions of various combinatorial objects including independent sets and matchings. A version of the local optimality of Gibbs distributions corresponding to independent sets (hard-core model) on regular trees is already addressed in [18], [11], and [19]. 
Proof of Theorem 2. Again we start by proving the result for $\mathcal{I}_{w}(n, r)$. The proofs for other three objects is similar and we highlight some differences in the end. Let $V_{r} \subset G_{r}(n)$ denote the independent set which achieves the maximum weight $\mathcal{I}_{w}(n, r)$. It is almost surely unique by the continuity of the distribution $F_{w}$. Consider a randomly selected node of the graph $G$, which, w.l.g., we may assume is node 0 . By symmetry we have $\mathbb{E}\left[\mathcal{I}_{w}(n, r)\right]=n \mathbb{E}\left[W_{0} 1\{0 \in\right.$ $\left.V_{r}\right\}$ ]. Let us fix a large positive integer $d$, which is a constant independent from $n$, and let $H(d)$ denote the depth- $d$ neighborhood of 0 . That is, $H$ is the collection of nodes in $G$ which are connected to 0 by paths with length $\leq d$. It is well known that, w.h.p. as $n \rightarrow \infty, H$ is a depth- $d r$-regular tree, [16], except in this case, unlike in Subsection 4.2, the root node has outdegree $r$ and the remaining nonleaf nodes have outdegree $r-1$. Let $\partial H$ denote the leaves of this tree (level $d$ ). Fix any binary vector $\bar{b}$ with dimension $|\partial H|+|G \backslash H|$, and any nonnegative vector $\bar{w}$ also with dimension $|\partial H|+|G \backslash H|$. Assume the vector $\bar{b}$ is such that if two nodes in $\partial H \cup(G \backslash H)$ are connected by an edge, only one of these two nodes can have the corresponding component of $\bar{b}$ equal to 1 . That is, the nodes marked 1 by $\bar{b}$ correspond to some independent set in $\partial H \cup(G \backslash H)$. Consider the problem of finding the maximum weight independent set $V_{r}$ in $G$ when the weights of the nodes in $\partial H \cup(G \backslash H)$ are conditioned to be $\bar{w}$, nodes $i \in \partial H \cup(G \backslash H)$ with the corresponding component of $\bar{b}$ equal to 1 are conditioned to belong to $V_{r}$, and the remaining nodes in $\partial H \cup(G \backslash H)$ are conditioned not to belong to $V_{r}$. Notationwise, we consider the value $\left(\mathcal{I}_{w}(n, r) \mid(\bar{b}, \bar{w})\right)$. In particular, we need to select to maximum weight independent set in the tree $H$, which is consistent with conditioning $(\bar{b}, \bar{w})$. Naturally, the consistency needs to be checked only across the boundary $\partial H$.

Let $(B \mid(\bar{b}, \bar{w}))$ and $\left(B_{i} \mid(\bar{b}, \bar{w})\right), 1 \leq i \leq r$, denote the bonus of the node 0 and the bonuses of its neighboring nodes $1,2, \ldots, r$, respectively. By Lemma 6 , we have $(B \mid(\bar{b}, \bar{w}))=$ $\max \left(0, W_{0}-\sum_{1 \leq i \leq r}\left(B_{i} \mid(\bar{b}, \bar{w})\right)\right)$. Since the distribution function $F_{w}$ of $W_{0}$ is continuous, then $W_{0}-\sum_{1 \leq i<r}\left(B_{i} \mid(\bar{b}, \bar{w})\right)=0$ with probability 0 . Then applying the second part of Lemma 6 , node 0 belongs to the maximum weight independent set if and only if $B=$ $W_{0}-\sum_{1 \leq i \leq r}\left(B_{i} \mid(\bar{b}, \bar{w})\right)>0$. Therefore,

$$
\frac{\mathbb{E}\left[\mathcal{I}_{w}(n, r)\right]}{n}=\int_{\bar{b}, \bar{w}} \mathbb{E}\left[W_{0} 1\left\{W_{0}-\sum_{1 \leq i \leq r}\left(B_{i} \mid(\bar{b}, \bar{w})\right)>0\right\}\right] d \mathbb{P}(\bar{b}, \bar{w}) .
$$

But by Theorem 8 , for every $\bar{b}, \bar{w}$ the distribution of $\left(B_{i} \mid(\bar{b}, \bar{w})\right)$ converges to $F^{*}$ uniformly in $\bar{b}, \bar{w}$ as $d$ becomes large. We conclude

$$
\lim _{n} \frac{\mathbb{E}\left[\mathcal{I}_{w}(n, r)\right]}{n}=\mathbb{E}\left[W_{0} 1\left\{W_{0}-\sum_{1 \leq i \leq r} B_{i}>0\right\}\right],
$$

where $B_{i} \sim F^{*}$. This completes the proof for the maximum weight independent set in $G_{r}(n)$. When the graph $G(n, c / n)$ is considered, the proof is very similar, we just use the fact that $H$ - depth $d$ neighborhood of 0 approaches in distribution a Poisson tree [22]. The proofs for maximum weight matchings are similar; we use Lemma 7 instead of Lemma 6.

Proof of Corollary 2. In fact we have proved this result already en route of proving Theorem 2 above. We have shown, that given $\bar{b}, \bar{w}$ and conditioned on $H$ being a tree, a fixed node 0 belongs to the maximum weight independent set almost surely iff $(B \mid(\bar{b}, \bar{w}))=W_{0}-\sum_{1 \leq i \leq r}\left(B_{i} \mid(\bar{b}, \bar{w})\right)>0$. Repeating the proof of Theorem 2 , $\mathcal{I N}_{w}(n, r) / n=\mathbb{E}\left[1\left\{0 \in V_{r}\right\}\right] \rightarrow \mathbb{E}\left[1\left\{W_{0}-\sum_{1 \leq i \leq r} B_{i}\right\}\right]$, where $B_{i} \sim F^{*}$. 
Proof of Theorem 4. The essential ingredients for the proof of this results are already established in the proof of Theorem 2 above. We start with $\mathcal{I}_{w}(n, r)$. The proofs for other cases are very similar and we omit them. Let $\mathcal{O}=\mathcal{O}(n, r) \subset[n]$ be the independent set achieving the maximum weight. Fix two nodes $i, j \in[n]$ and arbitrary $\varepsilon>0$. Let $H=H(i, d)$ denote the depth- $d$ graph-theoretic neighborhood of $i$ in the graph $G=G_{r}(n)$ and let $\partial H$ denote the boundary of $H$ - the nodes of $H$ at distance $d$ from $i$. Let $\mathcal{E}_{T}$ denote the event that $H$ is a ( $r$-regular) tree. From the theory of random regular graphs [16], $\mathbb{P}\left(\mathcal{E}_{T}\right) \rightarrow 1$ as $n \rightarrow \infty$. As a result $\mathbb{P}\left(\mathcal{E}_{T}\right) \geq 1-\varepsilon$ for all $n \geq n_{0}$ for some $n_{0}=n_{0}(d)$ (note the dependence on $d)$. Fix any realization of $(G \backslash H) \cup \partial H$ together with the realization of the weights $\bar{w}$ of nodes in $G \backslash(H \cup \partial H)$ and indicators $\bar{b}$ of whether the nodes belong to $\mathcal{O}$. As far as deciding which nodes of $H \backslash \partial H$ are in $\mathcal{O}$ and in particular whether node $i$ belongs to $\mathcal{O}$, only the restriction of $\bar{w}, \bar{b}$ to $\partial H$ is relevant. Conditioning on the event $\mathcal{E}_{T}$, denote by $(B \mid(\bar{b}, \bar{w}))$ the bonus of $i$ in $H$ [for completeness define $(B \mid(\bar{b}, \bar{w}))$ to be zero when the event $\mathcal{E}_{T}$ does not hold]. Applying Lemma 6 and the continuity of $F_{w}$, we have $i \in \mathcal{O}$ iff $(B \mid(\bar{b}, \bar{w}))>0$. Applying Theorem $8,|\mathbb{P}((B \mid(\bar{b}, \bar{w}))>0)-\mathbb{P}(B>0)|<\varepsilon$ for all $d \geq d_{0}(\varepsilon)$ for some $d_{0}(\varepsilon)$, and for any $\bar{w}, \bar{b}$, where $B \sim F^{*}$ and $F^{*}$ is the unique fixed point of $T^{2}=T_{\mathcal{I}, r-1}^{2}$. Thus

$$
\begin{aligned}
|\mathbb{P}(i \in \mathcal{O})-\mathbb{P}(B>0)| & \leq \int_{\hat{G} \bar{w}, \bar{b}}\left|\mathbb{P}\left(i \in \mathcal{O} \mid \mathcal{E}_{T}, \hat{G}, \bar{w}, \bar{b}\right)-\mathbb{P}(B>0)\right| d \mathbb{P}\left(\mathcal{E}_{T}, \hat{G}, \bar{w}, \bar{b}\right) \\
& +\left|\mathbb{P}\left(i \in \mathcal{O} \mid \overline{\mathcal{E}}_{T}\right)-\mathbb{P}(B>0)\right| \mathbb{P}\left(\overline{\mathcal{E}}_{T}\right) \\
& \leq 2 \varepsilon,
\end{aligned}
$$

whenever $d \geq d_{0}(\varepsilon)$ and $n \geq n_{0}(d)$, where $\hat{G}$ denotes a realization of the subgraph $(G \backslash$ $H) \cup \partial H$. Observe that since $|H| \leq 1+r+(r-1)^{2}+\cdots+(r-1)^{d}$ then $\mathbb{P}(j \notin H) \geq 1-\varepsilon$ for all $n \geq n_{1}(d)$ for some $n_{1}(d)$. Then

$$
\begin{aligned}
\mid \mathbb{P}(i, j \in \mathcal{O}) & -\mathbb{P}(i \in \mathcal{O}) \mathbb{P}(j \in \mathcal{O}) \mid \\
\leq & \left|\mathbb{P}\left(i \in \mathcal{O} \mid \mathcal{E}_{T}, j \notin H, j \in \mathcal{O}\right) \mathbb{P}\left(\mathcal{E}_{T}, j \notin H, j \in \mathcal{O}\right)-\mathbb{P}(i \in \mathcal{O}) \mathbb{P}(j \in \mathcal{O})\right| \\
& +\mathbb{P}\left(i \in \mathcal{O}, \mathcal{E}_{T}, j \in H \cap \mathcal{O}\right)+\mathbb{P}\left(i \in \mathcal{O}, \overline{\mathcal{E}}_{T}, j \in \mathcal{O}\right)
\end{aligned}
$$

The event $j \notin H, j \in \mathcal{O}$ is completely described by the realizations $\hat{G}, \bar{w}, \bar{b}$. Since $|\mathbb{P}((B \mid(\bar{b}, \bar{w}))>0)-\mathbb{P}(B>0)|<\varepsilon$ for all $d \geq d_{0}(\varepsilon)$, then

$$
\left|\mathbb{P}\left(i \in \mathcal{O} \mid \mathcal{E}_{T}, j \notin H, j \in \mathcal{O}\right)-\mathbb{P}(B>0)\right|<\varepsilon,
$$

for all $d \geq d_{0}(\varepsilon)$. Also $\mathbb{P}(j \in \mathcal{O})=\mathbb{P}\left(\mathcal{E}_{T}, j \notin H, j \in \mathcal{O}\right)+\mathbb{P}\left(\mathcal{E}_{T}, j \in H \cap \mathcal{O}\right)+\mathbb{P}\left(\overline{\mathcal{E}}_{T}, j \in \mathcal{O}\right)$. But $\mathbb{P}\left(\mathcal{E}_{T}, j \in H \cap \mathcal{O}\right) \leq \mathbb{P}(j \in H) \leq \varepsilon$ for all $n \geq n_{1}(d)$ and $\mathbb{P}\left(\overline{\mathcal{E}}_{T}, j \in \mathcal{O}\right) \leq \mathbb{P}\left(\overline{\mathcal{E}}_{T}\right) \leq \varepsilon$ for all $n \geq n_{0}(d)$. As a result

$$
\left|\mathbb{P}(j \in \mathcal{O})-\mathbb{P}\left(\mathcal{E}_{T}, j \notin H, j \in \mathcal{O}\right)\right| \leq 2 \varepsilon
$$

whenever $n \geq \max \left(n_{0}\left(d_{0}(\varepsilon)\right), n_{1}\left(d_{0}(\varepsilon)\right)\right)$. Combining (35), (32), and (36), we obtain

$$
\begin{aligned}
\mathbb{P}\left(i \in \mathcal{O} \mid \mathcal{E}_{T}, j \notin\right. & H, j \in \mathcal{O}) \mathbb{P}\left(\mathcal{E}_{T}, j \notin H, j \in \mathcal{O}\right)-\mathbb{P}(i \in \mathcal{O}) \mathbb{P}(j \in \mathcal{O}) \\
& \leq(\mathbb{P}(i \in \mathcal{O})+3 \varepsilon)(\mathbb{P}(j \in \mathcal{O})+2 \varepsilon)-\mathbb{P}(i \in \mathcal{O}) \mathbb{P}(j \in \mathcal{O}) \\
& \leq 5 \varepsilon+6 \varepsilon^{2}<6 \varepsilon
\end{aligned}
$$


Similarly we show

$$
\mathbb{P}\left(i \in \mathcal{O} \mid \mathcal{E}_{T}, j \notin H, j \in \mathcal{O}\right) \mathbb{P}\left(\mathcal{E}_{T}, j \notin H, j \in \mathcal{O}\right)-\mathbb{P}(i \in \mathcal{O}) \mathbb{P}(j \in \mathcal{O}) \geq-6 \varepsilon .
$$

We conclude that the value in (33) is bounded by $6 \varepsilon$. Each summand in (34) is bounded by $\varepsilon$ since $\mathbb{P}(j \in H)$ when $n \geq n_{1}\left(d_{0}(\varepsilon)\right)$ and $\mathbb{P}\left(\overline{\mathcal{E}}_{T}\right) \leq \varepsilon$ when $n \geq n_{0}\left(d_{0}(\varepsilon)\right)$. We conclude that whenever $n \geq \max \left(n_{0}\left(d_{0}(\varepsilon)\right), n_{1}\left(d_{0}(\varepsilon)\right)\right),|\mathbb{P}(i, j \in \mathcal{O})-\mathbb{P}(i \in \mathcal{O}) \mathbb{P}(j \in \mathcal{O})| \leq 8 \varepsilon$. This completes the proof of the theorem.

\subsection{Computation of Limits. Exponentially Distributed Weights}

We prove Theorem 3 in this subsection. With the help of Theorem 2, we can focus on proving the uniqueness and computing the fixed points of the operator $T^{2}$. As usual we start with maximum weight independent set in $G_{r}(n)$. The analysis of other cases is similar and will follow immediately. The calculations are similar to the ones in [5] performed for maximum weight matching in random trees. The difference is that we have to compute the fixed point of the operator $T^{2}$ and not just $T$.

\section{Proof of Theorem 3.}

- Independent sets in $G_{r}(n)$. Let $F^{*}$ denote any fixed point distribution of $T^{2}=T_{\mathcal{I}, r-1}^{2}$ (at least one exists by Proposition 1$)$, and let $B \sim F^{*}$. Then $B=\max (0, W-$ $\left.\sum_{1 \leq i \leq r-1} \hat{B}_{i}\right), W \sim \operatorname{Exp}(1), \hat{B}_{i} \sim T\left(F^{*}\right)$. Similarly, if $\hat{B} \sim T\left(F^{*}\right)$, then $\hat{B}=$ $\max \left(0, W-\sum_{1 \leq i \leq r-1} B_{i}\right), W \sim \operatorname{Exp}(1), B_{i} \sim F^{*}$. Then for any $t \geq 0$

$$
\mathbb{P}(B>t)=\mathbb{P}\left(W>\sum_{1 \leq i \leq r-1} \hat{B}_{i}+t\right)=e^{-t} \mathbb{P}\left(W>\sum_{1 \leq i \leq r-1} \hat{B}_{i}\right),
$$

and similarly

$$
\mathbb{P}(\hat{B}>t)=e^{-t} \mathbb{P}\left(W>\sum_{1 \leq i \leq r-1} B_{i}\right),
$$

where we use the memoryless property of the exponential distribution. Let $b=\mathbb{P}(B=$ $0), \hat{b}=\mathbb{P}(\hat{B}=0)$, where $B \sim F^{*}$ and $\hat{B} \sim T\left(F^{*}\right)$. Our next goal is computing $b$ and $\hat{b}$. From the above we obtain

$$
\mathbb{P}(B>t)=e^{-t}(1-b), \quad \mathbb{P}(\hat{B}>t)=e^{-t}(1-\hat{b}),
$$

implying

$$
\mathbb{P}(B>t \mid B>0) \sim \operatorname{Exp}(1), \quad \mathbb{P}(\hat{B}>t \mid \hat{B}>0) \sim \operatorname{Exp}(1) .
$$

Then

$$
b=\mathbb{P}\left(W-\sum_{1 \leq i \leq r-1} \hat{B}_{i} \leq 0\right)=\int_{0}^{\infty} e^{-t} \mathbb{P}\left(\sum_{1 \leq i \leq r-1} \hat{B}_{i} \geq t\right) d t
$$

In order to compute $\mathbb{P}\left(\sum_{1 \leq i \leq r-1} \hat{B}_{i} \geq t\right)$, we condition on $j \leq r-1$ terms $\hat{B}_{i}$ out of $r-1$ being equal to zero, and the rest positive. This occurs with probability 
$\left(\begin{array}{c}r-1 \\ j\end{array}\right) \hat{b}^{j}(1-\hat{b})^{r-1-j}$. When $j<r-1$, the sum of $r-1-j$ nonzero terms $\hat{B}_{i}$ has an Erlang distribution with parameter $r-1-j$ (sum of $r-1-j$ independent random variables distributed as $\operatorname{Exp}(1))$. The density function of this distribution is $f(z)=\frac{z^{r-2-j}}{(r-2-j) !} e^{-z}$ and the tail probability is

$$
\mathbb{P}(\cdot>t)=\int_{t}^{\infty} \frac{z^{r-2-j}}{(r-2-j) !} e^{-z} d z=\sum_{0 \leq i \leq r-2-j} \frac{t^{i}}{i !} e^{-t}
$$

and

$$
\int_{0}^{\infty} e^{-t} \mathbb{P}(\cdot>t) d t=\sum_{0 \leq i \leq r-2-j} \frac{1}{2^{i+1}}=1-\frac{1}{2^{r-1-j}}
$$

Combining with (39) and interchanging integration and summation, we obtain

$$
b=\sum_{0 \leq j \leq r-1}\left(\begin{array}{c}
r-1 \\
j
\end{array}\right) \hat{b}^{j}(1-\hat{b})^{r-1-j}\left(1-\frac{1}{2^{r-1-j}}\right)=1-\left(\frac{1+\hat{b}}{2}\right)^{r-1}
$$

Similar calculations lead to $\hat{b}=1-\left(\frac{1+b}{2}\right)^{r-1}$. Combining

$$
b=f(f(b)), \quad \text { where } f(x)=1-\left(\frac{1+x}{2}\right)^{r-1}
$$

Lemma 10. Equation (42) has a unique solution $b^{*}$ within the range $b \in[0,1]$ iff $r \leq 4$. In this case $b^{*}$ is also the unique solution of $f(b)=b$.

Figures 1 and 2 show the graphs of $f(f(b))$ for the cases $r=4$ and $r=8$. The first corresponds to the case of the unique solution. In the second case there is more than one solution.

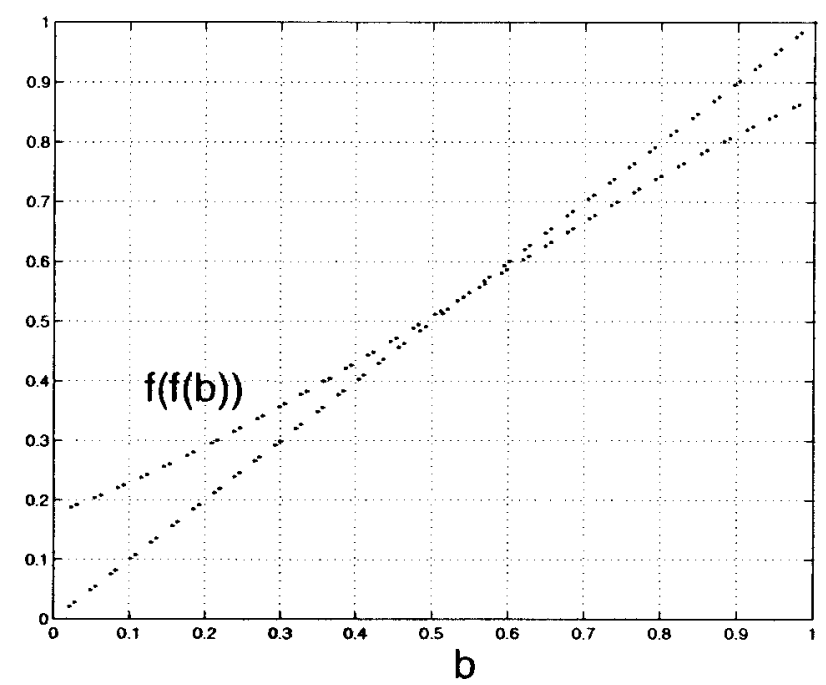

Fig. 1. $f(f(b))=b$ has one solution when $r=4$. 


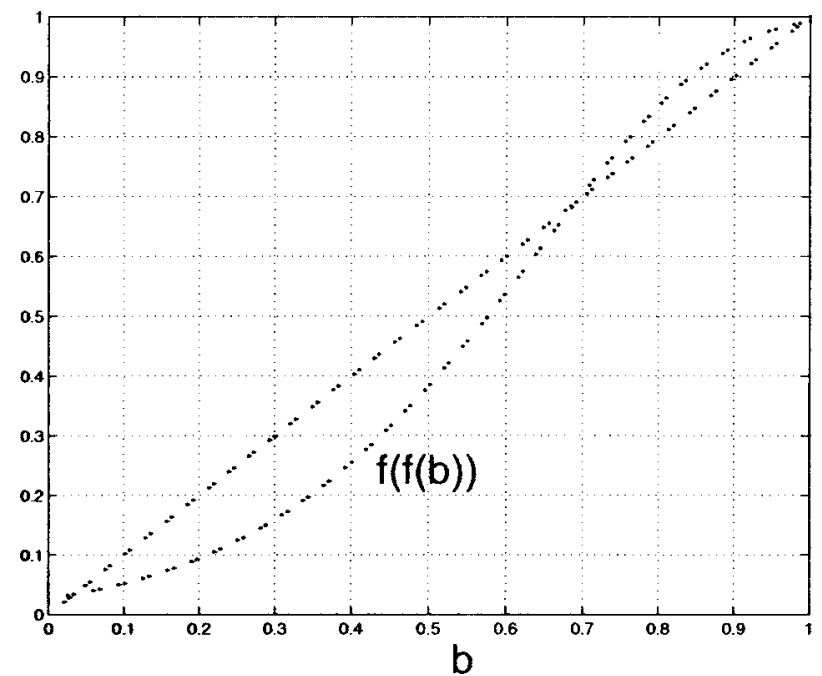

Fig. 2. $f(f(b))=b$ has more than one solution when $r=8$.

Proof. Note that for every $r \geq 1$ the equation $x=f(x)$ has exactly one solution in $[0,1]$ since $f$ is a strictly decreasing function and $f(0)=1-1 / 2^{r-1}>f(1)=0$. This solution is also a solution to $x=f(f(x))$.

We now prove the uniqueness for $2 \leq r \leq 4$ and nonuniqueness for $r>4$. Let $r \leq 4$. We claim that for all $x \in[0,1]$

$$
\frac{d f(f(x))}{d x}=\frac{(r-1)^{2}}{4}\left(\frac{1+f(x)}{2}\right)^{r-2}\left(\frac{1+x}{2}\right)^{r-2}<1 .
$$

This would imply that $f(f(x))-x$ is a strictly decreasing function and is equal to zero in at most one point. (43) is equivalent to

$$
(1+f(x))(1+x)<\frac{2^{\frac{2 r-2}{r-2}}}{(r-1)^{\frac{2}{r-2}}} .
$$

Let us check the validity of this inequality at the end points $x=0,1$. Since $f(0)<$ $1, f(1)=0$, the left-hand side in both points is at most 2 , which is strictly smaller than the right-hand side for $r=2,3,4$, as is easily checked. It remains to check the inequality at the points where the derivative of the function $g(x)=(1+f(x))(1+x)$ vanishes. We have

$$
\begin{aligned}
\dot{g}(x) & =1+\dot{f}(x)+f(x)+x \dot{f}(x) \\
& =1-\frac{r-1}{2}\left(\frac{1+x}{2}\right)^{r-2}+1-\left(\frac{1+x}{2}\right)^{r-1}-x \frac{r-1}{2}\left(\frac{1+x}{2}\right)^{r-2} \\
& =2-\left(\frac{1+x}{2}\right)^{r-1}-\frac{(1+x)(r-1)}{2}\left(\frac{1+x}{2}\right)^{r-2} \\
& =2-r\left(\frac{1+x}{2}\right)^{r-1} .
\end{aligned}
$$


Thus $\dot{g}(x)=0$ in exactly one point $x=2\left(\frac{2}{r}\right)^{\frac{1}{r-1}}-1$. We check that (44) holds in this point for $r=2,3,4$.

We now prove the nonuniqueness of the solution to (42) when $r \geq 5$. Let $b^{*}$ denote the unique fixed point of $f\left(b^{*}\right)=b^{*}$. Clearly $f\left(f\left(b^{*}\right)\right)=b^{*}$. We claim that

$$
\left.\frac{d f(f(x))}{d x}\right|_{x=b^{*}}>1
$$

This implies the result since we get that for $\varepsilon$ sufficiently small, $f\left(f\left(b^{*}-\varepsilon\right)\right)<b^{*}-\varepsilon$. But $f(f(0))>0$. Therefore, there exists a different fixed point of (42) in the interval $\left(0, b^{*}\right)$. To show (45), note that

$$
\left.\frac{d f(f(x))}{d x}\right|_{x=b^{*}}=\frac{(r-1)^{2}}{4}\left(\frac{1+f\left(b^{*}\right)}{2}\right)^{r-2}\left(\frac{1+b^{*}}{2}\right)^{r-2}=\frac{(r-1)^{2}}{4}\left(\frac{1+b^{*}}{2}\right)^{2 r-4} .
$$

We need to show that

$$
\frac{(r-1)^{2}}{4}\left(\frac{1+b^{*}}{2}\right)^{2 r-4}>1
$$

which is equivalent to $b^{*}>2\left(\frac{2}{r-1}\right)^{\frac{1}{r-2}}-1 \equiv b(r)$. We claim that $b(r)<f(b(r))=$ $1-\left(\frac{1+b(r)}{2}\right)^{r-1}$. Since $b^{*}=f\left(b^{*}\right)$ and $f$ is a decreasing function, this would imply $b(r)<b^{*}$ or (46). Note that $(1+b(r)) / 2=\left(\frac{2}{r-1}\right)^{\frac{1}{r-2}}$. Thus we need to check that

$$
\begin{aligned}
2\left(\frac{2}{r-1}\right)^{\frac{1}{r-2}}-1<1-\left(\frac{2}{r-1}\right)^{\frac{r-1}{r-2}} & \Leftrightarrow 2\left(\frac{2}{r-1}\right)^{\frac{1}{r-2}}<2-\left(\frac{2}{r-1}\right)^{\frac{r-1}{r-2}} \\
& \Leftrightarrow\left(\frac{2}{r-1}\right)^{\frac{1}{r-2}}<1-\frac{2^{\frac{1}{r-2}}}{(r-1)^{1+\frac{1}{r-2}}} \\
& =1-\left(\frac{1}{r-1}\right)\left(\frac{2}{r-1}\right)^{\frac{1}{r-2}} \\
& \Leftrightarrow\left(\frac{2}{r-1}\right)^{\frac{1}{r-2}}<\frac{r-1}{r} \\
& \Leftrightarrow 2<(r-1)\left(1-\frac{1}{r}\right)^{r-2} .
\end{aligned}
$$

Note that $g(r)=\left(1-\frac{1}{r}\right)^{r-2}$ is a strictly growing function of $r$ since $\log (g(r))=$ $(r-2) \log \left(1-\frac{1}{r}\right)$. Therefore, it suffices to verify that for $r=5$ we have $2<$ $4\left(1-\frac{1}{5}\right)^{3} \approx 2.048$.

Applying Lemma $10, b=\hat{b}$ is the unique solution of $f(b)=b$. When $r=2$, we obtain $b=1 / 3$. When $r=3$, we obtain $b=1-(1+b)^{2} / 4$ or $b=2 \sqrt{3}-3 \approx .4641$. When $r=4$, we find numerically that $b \approx .5419$.

Recall that the distribution $F^{*}$ of $B$ is given by $\mathbb{P}(B=0)=b$ and $\mathbb{P}(B>t)=$ $(1-b) e^{-t}$. Applying (7) of Theorem 2 and generating $B_{1}, \ldots, B_{r} \sim F^{*}$ independently, 
we obtain

$$
\lim _{n} \frac{\mathcal{I}_{w}(n, r)}{n}=\int_{0}^{\infty} t e^{-t} \mathbb{P}\left(t>\sum_{1 \leq i \leq r} B_{i}\right) d t
$$

We again condition on $j$ out of $r$ terms $B_{i}$ to be equal to zero and the rest positive. This occurs with probability $\left(\begin{array}{l}r \\ j\end{array}\right) b^{j}(1-b)^{r-j}$. W.l.g. assume that the nonzero terms are $B_{1}, \ldots, B_{r-j}$. We have

$$
\int_{0}^{\infty} t e^{-t} \mathbb{P}\left(t>\sum_{1 \leq i \leq r-j} B_{i}\right) d t=1-\int_{0}^{\infty} t e^{-t} \mathbb{P}\left(\sum_{1 \leq i \leq r-j} B_{i}>t\right) d t .
$$

Repeating the calculations (40), we obtain that $\mathbb{P}\left(\sum_{1 \leq i \leq r-j} B_{i}>t\right)=$ $\sum_{0 \leq i \leq r-1-j} \frac{t^{i}}{i !} e^{-t}$. Then the expression of the right-hand side of (48) becomes

$$
1-\int_{0}^{\infty} t e^{-t} \sum_{0 \leq i \leq r-1-j} \frac{t^{i}}{i !} e^{-t} d t=1-\sum_{0 \leq i \leq r-1-j} \frac{i+1}{2^{i+2}}=\frac{r-j+2}{2^{r-j+1}} .
$$

Combining, we obtain

$$
\begin{aligned}
\lim _{n} \frac{\mathcal{I}_{w}(n, r)}{n}= & \sum_{0 \leq j \leq r}\left(\begin{array}{l}
r \\
j
\end{array}\right) b^{j}(1-b)^{r-j} \frac{r-j+2}{2^{r-j+1}}=\frac{1}{2} 2 \sum_{0 \leq j \leq r}\left(\begin{array}{l}
r \\
j
\end{array}\right) b^{j}\left(\frac{1}{2}-\frac{b}{2}\right)^{r-j} \\
& +\frac{1}{2} \sum_{0 \leq j \leq r}\left(\begin{array}{l}
r \\
j
\end{array}\right) b^{j}\left(\frac{1}{2}-\frac{b}{2}\right)^{r-j}(r-j) .
\end{aligned}
$$

The first summand is simply $\left(\frac{1}{2}+\frac{b}{2}\right)^{r}$. We compute the second summand using the following probabilistic argument. Rewrite the expression as

$$
\left(\frac{1}{2}+\frac{b}{2}\right)^{r} \sum_{0 \leq j \leq r}\left(\begin{array}{l}
r \\
j
\end{array}\right) \frac{b^{j}\left(\frac{1}{2}-\frac{b}{2}\right)^{r-j}}{\left(\frac{1}{2}+\frac{b}{2}\right)^{r}}(r-j) .
$$

The sum above is simply the expected number of successes in $r$ Bernoulli trials with the probability of success equal to $\left(\frac{1}{2}-\frac{b}{2}\right) /\left(\frac{1}{2}+\frac{b}{2}\right)=(1-b) /(1+b)$. Namely, it is $r(1-b) /(1+b)$. We obtain

$$
\lim _{n} \frac{\mathcal{I}_{w}(n, r)}{n}=\left(\frac{1}{2}+\frac{b}{2}\right)^{r}\left(1+\frac{r(1-b)}{2+2 b}\right)=\frac{(1+b)^{r-1}}{2^{r+1}}(2+2 b+r-r b) .
$$

Recall from Lemma 10 that $(1+b)^{r-1} / 2^{r-1}=1-b$. This proves (12). Plugging the corresponding value of $b$ for $r=2,3,4$, we obtain $\lim _{n} \frac{\mathcal{I}_{w}(n, 2)}{n}=\frac{2}{3}, \lim _{n} \frac{\mathcal{I}_{w}(n, 3)}{n}=$ .6077 , and $\lim _{n} \frac{\mathcal{I}_{w}(n, 4)}{n}=.5632$. This concludes the proofs for the case of maximum weight independent set in $G_{3}(n)$ and $G_{4}(n)$. Before we continue with other cases, it is convenient to prove Corollary 4, as the proof is almost immediate from above.

Proof of Corollary 4. We have established above that $T_{\mathcal{I}, r}^{2}$ has a unique fixed point iff $r \leq 4$. Applying (10), we need to compute $\mathbb{E}\left[1\left\{W-\sum_{1 \leq i \leq r} B_{i}>0\right\}\right]=\mathbb{P}(W>$ 
$\left.\sum_{1 \leq i \leq r} B_{i}\right)$, where $W \sim \operatorname{Exp}(1), B_{i} \sim F^{*}$. Instead of computing this quantity directly, note that the probability above is exactly $1-b$, if we the summation above was up to $r-1$ not $r$. Repeating the computations up to (41), we obtain

$$
\mathbb{P}\left(W>\sum_{1 \leq i \leq r} B_{i}\right)=\left(\frac{1+b}{2}\right)^{r} .
$$

Plugging the obtained values of $b$ for $r=2,3,4$, we obtain (19).

- Independent sets in $G(n, c / n)$. Let $F^{*}$ denote now any fixed point of $T^{2}=T_{\mathcal{I}, c}^{2}$. We introduce again $b=F^{*}(0)=\mathbb{P}\left(W-\sum_{1 \leq i \leq m} \hat{B}_{i} \leq 0\right)$, where $\hat{B}_{i} \sim T\left(F^{*}\right)$ and $m \sim$ Pois $(c)$. Similarly, $\hat{b}=T\left(F^{*}\right)(0)=\mathbb{P}\left(W-\sum_{1 \leq i \leq m} B_{i} \leq 0\right)$, where $B_{i} \sim F^{*}$. Repeating the computations done for $G_{r}(n)$, we obtain similarly to (41) that conditioning on $m=k$

$$
b=1-\left(\frac{1+\hat{b}}{2}\right)^{k} .
$$

Then $b=\sum_{k \geq 0} \frac{c^{k}}{k !} e^{-c}\left(1-\left(\frac{1+\hat{b}}{2}\right)^{k}\right)=1-e^{-\frac{c(1-\hat{b})}{2}}$. Similarly, $\hat{b}=1-e^{-\frac{c(1-b)}{2}}$. Thus, $b$ must satisfy $1-b=\exp \left(-\frac{c}{2} \exp \left(-\frac{c}{2}(1-b)\right)\right)$. Recall, however, that, by the second part of Theorem 1, the equation above has a unique solution iff $c \leq 2 e$. In this case $b$ is also the unique solution of $1-b=\exp \left(-\frac{c}{2}(1-b)\right)$. We now apply (7) of Theorem 2 to compute $\lim _{n} \frac{\mathcal{I}_{w}(n, c)}{n}$, where we substitute $m \sim \operatorname{Pois}(c)$ for $r$. In order to shortcut the computations, we use (47) and (50). We have

$$
\lim _{n} \frac{\mathcal{I}_{w}(n, c)}{n}=\sum_{k \geq 0} \frac{c^{k}}{k !} e^{-c} \int_{0}^{\infty} t e^{-t} \mathbb{P}\left(t>\sum_{1 \leq i \leq k} B_{i}\right) d t,
$$

where $B_{i} \sim F^{*}$. Recall, though, from (50), that

$$
\int_{0}^{\infty} t e^{-t} \mathbb{P}\left(t>\sum_{1 \leq i \leq k} B_{i}\right) d t=\left(\frac{1+b}{2}\right)^{k}\left(1+\frac{k(1-b)}{2(1+b)}\right) .
$$

Combining,

$$
\lim _{n} \frac{\mathcal{I}_{w}(n, c)}{n}=e^{-\frac{c(1-b)}{2}}+\frac{(1-b)}{2(1+b)} \frac{c(1+b)}{2} e^{-\frac{c(1-b)}{2}}=\left(1+\frac{c(1-b)}{4}\right) e^{-\frac{c(1-b)}{2}} .
$$

Using $b=1-\exp \left(-\frac{c(1-b)}{2}\right)$, we obtain (14).

- Matchings in $G_{r}(n)$. Let $F^{*}$ denote any fixed point distribution of $T=T_{\mathcal{M}, r}$. Then we have the following distributional identities: $\hat{B}=\max _{1 \leq i \leq r-1}\left(0, W_{i}-B_{i}\right)$, $W_{i} \sim \operatorname{Exp}(1), B_{i} \sim F^{*}, \hat{B} \sim T\left(F^{*}\right)$, and $B=\max _{1 \leq i \leq r-1}\left(0, W_{i}-\hat{B}_{i}\right), W_{i} \sim \operatorname{Exp}(1)$, $\hat{B}_{i} \sim T\left(F^{*}\right), B \sim F^{*}$. Let $\hat{b}=\mathbb{P}(W-\hat{B}<0)$, where $W \sim \operatorname{Exp}(1)$ and $\hat{B} \sim T\left(F^{*}\right)$, and let $b=\mathbb{P}(W-B<0)$, where $B \sim F^{*}$. Then for any $t \geq 0$

$$
\begin{aligned}
\mathbb{P}(B \leq t) & =\mathbb{P}\left(\max _{1 \leq i \leq r-1}\left(W_{i}-\hat{B}_{i}\right) \leq t\right)=\left(1-\mathbb{P}\left(W_{1}>t+\hat{B}_{1}\right)\right)^{r-1} \\
& =\left(1-e^{-t}(1-\hat{b})\right)^{r-1} .
\end{aligned}
$$


In particular

$$
\mathbb{P}(B=0)=\hat{b}^{r-1}, d \mathbb{P}(B \leq t)=(r-1)(1-\hat{b}) e^{-t}\left(1-e^{-t}(1-\hat{b})\right)^{r-2} d t, t>0 .
$$

(We note as above that $\mathbb{P}\left(W_{1}=\cdot\right)=0$, since $W_{1}$ has a continuous distribution.) Then

$$
\begin{aligned}
b & =\int_{0}^{\infty} e^{-t} \mathbb{P}(B>t) d t \\
& =\int_{0}^{\infty} e^{-t}\left(1-\left(1-e^{-t}(1-\hat{b})\right)^{r-1}\right) d t \\
& =1-\int_{0}^{\infty} e^{-t}\left(1-e^{-t}(1-\hat{b})\right)^{r-1} d t \\
& =1-\int_{0}^{\infty}\left(1-e^{-t}(1-\hat{b})\right)^{r-1} d\left(-e^{-t}\right) \\
& =1-\int_{0}^{1}(1-z(1-\hat{b}))^{r-1} d z \\
& =1-\left.\frac{(1-z(1-\hat{b}))^{r}}{r(-(1-\hat{b}))}\right|_{0} ^{1} \\
& =1-\frac{1-\hat{b}^{r}}{r(1-\hat{b})} .
\end{aligned}
$$

Similarly, we obtain

$$
\hat{b}=1-\frac{1-b^{r}}{r(1-b)},
$$

and combining we conclude that $b$ must be a fixed point of the equation $f(f(x))=x$, where

$$
f(x)=1-\frac{1-x^{r}}{r(1-x)}
$$

Lemma 11. For every $r \geq 1$ the equation $f(f(x))=x$ has a unique solution $x^{*}$ in the range $[0,1]$, which is the unique solution of the equation $f(x)=x$.

Proof. Note that $\left(1-x^{r}\right) /(1-x)=1+x+\cdots+x^{r-1}$ and therefore $f(x)$ is a strictly decreasing function with $f(0)=1-1 / r, f(1)=0$. Therefore, $f(x)=x$ has exactly one solution $x^{*}$. We now prove that $f(f(x))>x$ for all $x<x^{*}$ and $f(f(x))<x$ for all $x>x^{*}$. This would complete the proof of the lemma. We need to show that for $x<x^{*}$

$$
f(f(x))=1-\frac{1-f^{r}(x)}{r(1-f(x))}>x,
$$

which is equivalent to $1-f^{r}(x)<r(1-f(x))(1-x)$. But since $f$ is a decreasing function and $f\left(x^{*}\right)=x^{*}$, then $f(x)>x$ for all $x<x^{*}$ and therefore $1-f^{r}(x)<$ 
$1-x^{r}=r(1-f(x))(1-x)$. Similarly, when $x>x^{*}$, we have $f(x)<x^{*}$, and then $1-f^{r}(x)>1-x^{r}=r(1-f(x))(1-x)$, resulting in

$$
f(f(x))=1-\frac{1-f^{r}(x)}{r(1-f(x))}<x .
$$

We conclude that $b=\hat{b}$ is determined as the unique solution of

$$
b=1-\frac{1-b^{r}}{r(1-b)}
$$

and the unique fixed point of $T^{2}$ is the distribution given by (51) with $b=\hat{b}$ given above. Now, using (8) of Theorem 2, (52), and adopting a convention $\left.\max _{2 \leq j \leq r}\left(W_{j}-B_{j}\right)\right)=0$ when $r=1$, we have

$$
\begin{aligned}
\lim _{n} \frac{\mathcal{M}_{w}(n, r)}{n} & =\frac{1}{2} \mathbb{E}\left[\sum_{1 \leq i \leq r} W_{i} 1\left\{W_{i}-B_{i}=\max _{1 \leq j \leq r}\left(0, W_{j}-B_{j}\right)\right\}\right] \\
& =\frac{r}{2} \mathbb{E}\left[W_{1} 1\left\{W_{1}-B_{1}>\max _{2 \leq j \leq r}\left(0, W_{j}-B_{j}\right)\right\}\right] \\
& =\frac{r}{2} \int_{0}^{\infty} t e^{-t} b^{r-1} \mathbb{P}\left(t>\max _{2 \leq j \leq r}\left(W_{j}-B_{j}\right)\right) d t \\
& +\frac{r}{2} \int_{0}^{\infty} \int_{0}^{t} t e^{-t}(r-1)(1-b) \\
& \times e^{-z}\left(1-e^{-z}(1-b)\right)^{r-2} \mathbb{P}\left(t-z>\max _{2 \leq j \leq r}\left(W_{j}-B_{j}\right)\right) d t d z,
\end{aligned}
$$

where the summands (55) and (56) corresponds to conditioning on $B_{1}=0$ and $B_{1}=$ $z>0$.

We now compute the integrals in these summands. We have

$$
\begin{aligned}
\int_{0}^{\infty} t e^{-t} b^{r-1} \mathbb{P}\left(t>\max _{2 \leq j \leq r}\left(W_{j}-B_{j}\right)\right) d t & =\int_{0}^{\infty} t e^{-t} b^{r-1} \mathbb{P}^{r-1}\left(t>W_{2}-B_{2}\right) d t \\
& =\int_{0}^{\infty} t e^{-t} b^{r-1}\left(1-\mathbb{P}\left(W_{2}>t+B_{2}\right)\right)^{r-1} d t \\
& =\int_{0}^{\infty} t e^{-t} b^{r-1}\left(1-e^{-t} \mathbb{P}\left(W_{2}>B_{2}\right)\right)^{r-1} d t \\
& \left.=\int_{0}^{\infty} t e^{-t} b^{r-1}\left(1-e^{-t}(1-b)\right)\right)^{r-1} d t .
\end{aligned}
$$

Similarly, we obtain that the integral in (56) is equal to

$$
\int_{0}^{\infty} \int_{0}^{t} t e^{-t}(r-1)(1-b) e^{-z}\left(1-e^{-z}(1-b)\right)^{r-2}\left(1-e^{-t+z}(1-b)\right)^{r-1} d t d z
$$

Substituting to summands in (55) and (56), we obtain (15). 
- Matchings in $G(n, c / n)$. The derivation is very similar to the one for $\mathcal{M}_{w}(n, r)$. We introduce $b$ and $\hat{b}$ exactly as above. Equation (53) becomes

$$
\begin{aligned}
b & =\sum_{m \geq 0} \frac{c^{m}}{m !} e^{-c}\left(1-\frac{1-\hat{b}^{m+1}}{(m+1)(1-\hat{b})}\right) \\
& =1-\frac{1}{c(1-\hat{b})} \sum_{m \geq 0} \frac{c^{m+1}}{(m+1) !} e^{-c}+\frac{1}{c(1-\hat{b})} \sum_{m \geq 0} \frac{c^{m+1} \hat{b}^{m+1}}{(m+1) !} e^{-c} \\
& =1-\frac{1}{c(1-\hat{b})}+\frac{e^{-c(1-\hat{b})}}{c(1-\hat{b})},
\end{aligned}
$$

Then $1-b$ is a fixed point of the equation $f(f(x))=x$, where $f(x)=\frac{1-e^{-c x}}{c x}$. That is

$$
\frac{1-e^{c^{\frac{1-e^{-c x}}{c x}-c}}}{c^{\frac{1-e^{-c x}}{c x}}}=x
$$

First we note that $x=0$ does not satisfy $f(f(x))=x$, so we assume $x>0$. The expression above then becomes $e^{-c x}+c x^{2}-1=0$. The function $e^{-c x}+c x^{2}-1$ is a strictly convex function which is equal to zero at $x=0$, has derivative $-c<0$ at $x=0$, and diverges to infinity as $x$ diverges to infinity. Therefore, its graph has exactly one intersection with the horizontal axis in $x \in(0, \infty)$. Note that at $x=1$ the value is $e^{-c}+c-1>0$ (for every $c>0$ ); therefore there exists exactly solution to $e^{-c x}+c x^{2}-1=0$ in $x \in(0,1)$. We conclude that $b$ is uniquely determined by the equation $e^{-c(1-b)}+c(1-b)^{2}-1=0$. The remainder of the calculation is done just like for $G_{r}(n)$ by conditioning first on specific values of $r$ and recalling $m=r$ with probability $\frac{c^{r}}{r} ! e^{-c}$. We omit the fairly straightforward calculations.

Proof of Corollary 3. The result is essentially established en route of proving Theorems 2 and 3. Recall that in the proof of Theorem 2 the only place we used the randomness of our regular graph $G_{r}(n)$ was to say that a constant depth- $d$ neighborhood $H(d)$ of a randomly selected node $i \in[n]$ is a depth- $d r$-regular tree w.h.p. In the case of a cycle any constant depth- $d$ neighborhood of any node is w.p. 1 a path of length $2 d$ with the selected node in the middle. This is depth- $d$ 2-regular tree. The answer for the maximum weight matching on a cycle is the same as for independent set since we may simply assume the weights are assigned to nodes which are to the left for each edge.

\subsection{Deterministic and Bernoulli Weights}

Are the results obtained above relevant to the case when the weight of each node and edge $W=1$ with probability 1 or, in general, when the weights take some discrete values? Let us examine these questions with respect to our usual four operators $T$. We start with the case $W=1$. For $T=T_{\mathcal{I}, r}$, the corresponding distributional equation is $B=\max (0,1-$ $\left.\sum_{1 \leq i \leq r-1} B_{i}\right)$. If $B_{i}=0$, w.p.1, then $B=1$, w.p.1, and vice verse. Thus $B=0$ and $B=1$ are two fixed points of $T^{2}$, and $T^{2}$ does not have a unique fixed point distribution. There is a 
natural explanation for the lack of uniqueness, coming directly from the lack of long-range independence. Given a depth- $d r$-regular tree $T$ with all the weights equal to unity, note that the boundary does carry a nonvanishing information about the root in the following sense. If all the leaves of the tree (boundary nodes) are conditioned to belong to the maximum weight independent set, then all the parents of leaves cannot be part of the set. Then the maximum independent set is obtained by selecting all the nodes in level $d$, not selecting nodes in level $d-1$, selecting all the nodes in level $d-2$, and so on. In the end whether the root is selected is fully determined by the parity of $d$. Thereby, we do not have a long-range independence. Contrast this with the discussion in Brightwell and Winkler [11], where similar observation is used to show long-range dependence for Gibbs measures on infinite regular trees for the hard-core model. It is not hard to see that the similar lack of long-range independence holds for maximum weight matchings, where the weights are all 1.

The situation is different for $T=T_{\mathcal{I}, c}$. Let $F$ be a distribution function given by $F(t)=p, t \in[0,1), F(1)=1$. Namely, $F$ is simply a Bernoulli distribution with parameter $p(\operatorname{Be}(p))$. If $B=\max \left(0,1-\sum_{1 \leq i \leq m} B_{i}\right)$, where $B_{i} \sim F$ and $m \sim$ Pois $(c)$, then $B=1$ if $\sum_{1 \leq i \leq m} B_{i}=0$, which occurs with probability $\sum_{k \geq 0} \frac{c^{k}}{k !} e^{-c} p^{k}=e^{-c(1-p)}$, and $B=0$ otherwise. Thus $T(F)$ is $\operatorname{Be}\left(p_{1}\right)$ where $p_{1}=1-e^{-c(1-p)}$. Similarly $T^{2}(F)$ is $\operatorname{Be}\left(p_{2}\right)$, with $p_{2}=1-e^{-c e^{-c(1-p)}}$. In general, for $s=1,2, \ldots, T^{2 s}(F)$ is $\operatorname{Be}\left(p_{2 s}\right)$ with $1-p_{2 s}=e^{-c e^{-c\left(1-p_{2 s-2}\right)}}$. By Proposition 1 we know that for $F=\operatorname{Be}(0)$ and $F=\operatorname{Be}(1)$, $T^{2 s}(F)$ converges to some fixed point distributions $F_{* *}, F^{* *}$ which by the argument above are $\operatorname{Be}\left(p_{* *}\right), \operatorname{Be}\left(p^{* *}\right)$ with both $p=p_{* *}$ and $p=p^{* *}$ satisfying $1-p=e^{-c e^{-c(1-p)}}$. Recall from Theorem 1, that the equation $x=e^{-c e^{-c x}}$ has a unique solution iff $c \leq e$. By Proposition 1 this implies that when $c \leq e, T^{s}\left(F_{0}\right)$ converges to $\mathrm{Be}\left(p^{*}\right)$ for any starting distribution $F_{0}$. Again applying Proposition 1, we obtain that $T^{s}\left(F_{0}\right)$ converges to $\operatorname{Be}\left(p^{*}\right), T$ and $T^{2}$ have the same unique fixed point distribution $-\operatorname{Be}\left(p^{*}\right)$, where $p^{*}$ is also the unique solution of $1-p^{*}=e^{-c\left(1-p^{*}\right)}$.

It is a simple exercise to see that the same holds for $T=T_{\mathcal{M}, c}: T^{2}$ has a unique fixed point iff $c \leq e$, in which case the fixed point distribution is also $\operatorname{Be}\left(p^{*}\right)$. This is, of course, fully consistent with Theorem 1 . We summarize these observations.

Proposition 2. Let $F_{w}=1$. For every $r \geq 2, T_{\mathcal{I}, r}^{2}, T_{\mathcal{M}, r}^{2}$ have at least two fixed point distributions. $T_{\mathcal{I}, c}^{2}, T_{\mathcal{M}, c}^{2}$ have the unique fixed point distribution iff $c \leq e$ in which case the unique fixed point distribution is Be $\left(p^{*}\right)$, where $p^{*}$ is the unique solution of $1-p^{*}=e^{-c\left(1-p^{*}\right)}$.

Can we fully reproduce Theorem 1 for the case $c \leq e$ ? The problem with the case $F_{w}=1$ as, generally, with noncontinuous distributions $F_{w}$, is that the probability of $W-\sum_{1 \leq i \leq r} B_{i}=$ 0 is no longer zero. As a result, we do not have an exact condition purely in terms of $B=\max \left(0, W-\sum_{1 \leq i<r} B_{i}\right)$ for determining whether the root node belongs to say maximum weight independent set. One natural approach would be to approximate $F_{w}$ with a continuous distribution. But the difficulty is the lack of closed form expression for the solution of fixed point of $T^{2}\left(F^{*}\right)=F^{*}$. Such a solution $F^{*}$ can, though, be approximated numerically by computing $T^{2 s}(0)$ and $T^{2 s}\left(F_{w}\right)\left(T^{2 s}\left(F_{w, r}\right), T^{2 s}\left(F_{w, c}\right)\right)$ for matching) for large $s$ such that the differences between the two distributions is sufficiently small.

Suppose now the weights $W_{i}$ of the nodes are distributed as $\operatorname{Be}(z)$ for some parameter $z \in[0,1]$. For simplicity we will only consider the case $r=3$ and $T=T_{\mathcal{I}, 3}$ and obtain a complete criteria for uniqueness. 
Proposition 3. For $r=3$ the operator $T_{\mathcal{I}, r-1}^{2}$ has a unique fixed point distribution iff $z \in\left[\frac{1}{4}, 1\right]$. In this case the fixed point distribution is $B e(p)$ with $p=\frac{\sqrt{5-4 z}-1}{2(1-z)}$.

Proof. Let $F=\operatorname{Be}(p)$ for any $x \in[0,1]$. Then for $B^{\prime} \sim T(F)$ we have $B^{\prime}=\max (0, W-$ $\left.B_{1}-B_{2}\right)$, where $B_{1}, B_{2}$ are independent and distributed as $F$. Then $B^{\prime}=0$ when $W=0$ or when $W=1$ and $B_{1}+B_{2}>0$. This occurs with probability $z+(1-z)\left(1-p^{2}\right)=$ $1-(1-z) p^{2}$. Thus $B^{\prime} \sim \operatorname{Be}\left(1-(1-z) p^{2}\right)$. Repeating the development above for the case of the deterministic weight, we need to analyze the number of the solutions to the equation $f(f(x))=x$, where $f(x)=1-(1-z) x^{2}$. First, the equation $f(x)=x$ leads to the unique solution $x^{*}=\frac{-1+\sqrt{5-4 z}}{2(1-z)}$ (it is simple to check that the solution is in $[0,1]$ for every $z \in[0,1])$. Also $x^{*}$ is a solution of $g(x) \equiv f(f(x))=x$. We need to show that this is the unique solution of this equation iff $z>1 / 4$.

First we show that when $z<1 / 4, \dot{g}\left(x^{*}\right)>1$. Since $g(0)>0$, this would imply that there exists a solution of $g(x)=x$ in the open interval $\left(0, x^{*}\right)$ and the case $z<1 / 4$ would be resolved. We have $\dot{g}\left(x^{*}\right)=4(1-z)^{2} f\left(x^{*}\right) x^{*}=\left(2(1-z) x^{*}\right)^{2}$, where we use $f\left(x^{*}\right)=x^{*}$. Then the inequality $\dot{g}\left(x^{*}\right)>1$ holds when $x^{*}>1 /(2-2 z)$ which after as simple algebra is reduced to $z<1 / 4$. Thus when $z<1 / 4$ there are more than one fixed points of $T^{2}=T_{\mathcal{I}, 2}^{2}$.

Suppose now $z \geq 1 / 4$. Let again $x^{*}$ be the unique solution to $f\left(x^{*}\right)=x^{*}$ and consider $\dot{g}(x)=4(1-z)^{2} f(x) x$. We claim that when $z>1 / 4, \dot{g}(x)<1$ for all $x \in[0,1]$ and when $z=1 / 4, \dot{g}(x)<1$ for all $x \neq x^{*}$ and $\dot{g}\left(x^{*}\right)=1$. This immediately implies that $g(x)=x$ has at most one solution, meaning it has exactly one since $g\left(x^{*}\right)=x^{*}$. Note $\dot{g}(0)=0<1$. We now prove that $\dot{g}(1)=4(1-z)^{2} z<1$ and $\dot{g}(x)<1$ for all $x$ such that $\frac{d^{2} g(x)}{d x^{2}}=0$, except for $x=x^{*}$ for which we will show that $\dot{g}\left(x^{*}\right)=1$.

We start with $\dot{g}(1)=4(1-z)^{2} z \equiv \phi(z)$. Note $\phi(0)=\phi(1)=0<1$. Then the maximum is achieved at the points $z$ where $\dot{\phi}(z)=-8(1-z) z+4(1-z)^{2}=4(1-z)(1-3 z)=0$. We have already considered the case $z=1$. Otherwise $z=1 / 3$ for which $\phi(1 / 3)=16 / 27<1$. Thus $\dot{g}(1) \leq \sup _{z \in[0,1]} \phi(z)<1$.

Consider now points $x$ such that $\frac{d^{2} g(x)}{d x^{2}}=4(1-z)^{2}\left[-2(1-z) x^{2}+1-(1-z) x^{2}\right]=0$, from which we obtain $x=1 / \sqrt{3-3 z}$. Plugging this into

$$
\dot{g}(x)=4(1-z)^{2}\left(1-(1-z) x^{2}\right) x=4(1-z)^{2} \frac{2}{3} \frac{1}{\sqrt{3-3 z}}=\left(\frac{64(1-z)^{3}}{27}\right)^{\frac{1}{2}} .
$$

The last expression is smaller than unity whenever $z>1 / 4$. When $z=1 / 4$ the expression is equal to the unity. In this case, however, $x=1 / \sqrt{3-3 z}=2 / 3$ which is checked to be equal to $x^{*}=\frac{-1+\sqrt{5-4 z}}{2(1-z)}$ when $z=1 / 4$. This concludes the proof of the proposition.

\section{CONCLUSIONS}

We have derived in this paper the limits of maximum weight independent sets and matchings in sparse random graphs for some types of i.i.d weight distributions. Our method is based on a certain local optimality property, which states loosely that, in cases of certain distributions of the random weights, the optimal random combinatorial structure under the consideration exhibits a long-range independence and, as a result, the value which each node (edge) "contributes" to the optimal structure is almost completely determined by the constant depth neighborhood of the node (edge). We certainly believe that such local optimality 
holds for many other random combinatorial structures, and it seems to be an interesting property to study by itself, not to mention its applications to studying random combinatorial structures.

\section{ACKNOWLEDGMENTS}

The authors gratefully acknowledges interesting and fruitful discussions with David Aldous, Antar Bandyopadhyay, Yuval Peres, and Alan Frieze. The authors also wish to thank Antar Bandyopadhyay for correcting an earlier version of the manuscript, and Nick Wormald for informing the authors on the state of the art results on largest independent sets in sparse graphs. Finally, the authors wish to thank anonymous referees for excellent job in proofreading the manuscript and pointing the authors to several errors in the earlier version of the manuscript.

\section{REFERENCES}

[1] D. Aldous, Asymptotics in the random assignment problem, Probab Theory Related Fields 93 (1992), 507-534.

[2] D. Aldous, The $\zeta$ (2) limit in the random assignment problem, Random Structures Algorithms 18 (2001), 381-418.

[3] D. Aldous, Some open problems, http://stat-www.berkeley.edu/users/aldous/Research/ problems.ps.

[4] D. Aldous and A. Bandyopadhyay, A survey of max-type recursive distributional equations, Ann Appl Probab, to appear.

[5] D. Aldous and J. M. Steele, "The objective method: Probabilistic combinatorial optimization and local weak convergence," Discrete combinatorial probability, H. Kesten, (Editor), Springer, New York, 2003, pp. 1-72.

[6] J. Aronson, B. Pittel, and A. Frieze, Maximum matchings in sparse random graphs: Karp-Sipser revisited, Random Structures Algorithms 12 (1998), 11-178.

[7] A. Bandyopadhyay, Bivariate uniqueness in the logistic fixed point equation, Technical Report 629, Department of Statistics, University of California, Berkeley, 2002.

[8] A. Bandyopadhyay, Max-type recursive distributional equations, Ph.D. thesis, University of California, Berkeley, 2003.

[9] B. Bollabas, A probabilistic proof of an asymptotic formula for the number of regular graphs, European J Combinat 1 (1980), 311-316.

[10] B. Bollabas, The independence radio of regular graphs, Proc Amer Math Soc 83(2) (1981), 433-436.

[11] G. R. Brightwell and P. Winkler, Gibbs extremality for the hard-care model on a Bethe lattice, preprint, 2003.

[12] R. Durrett, Probability: Theory and examples, 2nd edition, Duxbury Press, Belmont, 1996.

[13] A. Frieze and S. Suen, On the independence number of random cubic graphs, Random Structures Algorithms 5 (1994), 649-664.

[14] D. Gamarnik, Linear phase transition in random linear constraint satisfaction problems, Probab Theory Related Fields. 129(3) (2004), 410-440.

[15] A. K. Hartmann and M. Weigt, Statistical mechanics perspective on the phase transition of vertex covering of finite-connectivity random graphs, Theoret Comput Sci 265 (2001), 199225 . 
[16] S. Janson, T. Łuczak, and A. Rucinske, Random graphs, Wiley New York, 2000.

[17] R. Karp and M. Sipser, Maximum matchings in sparse random graphs, 22nd Annu Symp Foundations of Computer Science, 1981, pp. 364-375.

[18] F. Kelly, Stochastic models of computer communication systems, J Roy Statist Soc Ser B 47(3) (1985), 379-395.

[19] J. Martin, Reconstruction thresholds on regular trees, Discrete Random Walks, DRW'03, C. Banderier and C. Krattenthaler, (Editors), Discrete Mathematics and Theoretical Computer Science Proceedings AC, DMTCS, Nancy, France, 2003, pp. 191-203.

[20] E. Mossel, Survey: Information flow on trees. Graphs, morphisms and statistical physiscs, J. Nestril and P. Winkler, (Editors), DIMACS Series in Discrete Mathematics and Theoretical Computer Science, American Mathematical Society, Providence, RI, 2004, pp. 155-170.

[21] U. A. Rozikov and Y. M. Suhov, A hard-core model on a Cayley tree: An example of a loss network, preprint, 2003.

[22] Joel H. Spencer, Ten lectures on the probabilistic method, 2nd edition, SIAM, Philadelphia, 1994.

[23] J. M. Steele, "Minimal spanning trees for graphs with random edge lenghts," Mathematics and computer science II. Algorithms, trees, combinatorics and probabilities, B. Chauvin, Ph. Faljolet, D. Gardy, and A. Mokkadem, (Editors), Birkhäuser, Boston, 2002, pp. 223-245.

[24] M. Talagrand, An assignment problem at high temperature, Ann Probab 31(2) (2003), 818-848.

[25] N. Wormald, Differential equations for random processes and random graphs, Ann Appl Probab 5 (1995), 1217-1235. 